\title{
Neutral Helium Spectral Lines in Dense Plasmas
}

\author{
Banaz Omar ${ }^{1}$, Sibylle Günter ${ }^{2}$, August Wierling ${ }^{1}$, and Gerd Röpke ${ }^{1}$ \\ ${ }^{1}$ Universität Rostock, Institut für Physik, \\ 18051 Rostock, Germany \\ ${ }^{2}$ Max-Planck-Institut für Plasmaphysik, \\ 85748 Garching, Germany
}

(Dated: February 9, 2007)

\begin{abstract}
Shift and broadening of isolated neutral helium lines $7281 \AA\left(2^{1} P-3^{1} S\right), 7065 \AA\left(2^{3} P-3^{3} S\right)$, $6678 \AA\left(2^{1} P-3^{1} D\right), 5048 \AA\left(2^{1} P-4^{1} S\right), 4922 \AA\left(2^{1} P-4^{1} D\right)$ and $4713 \AA\left(2^{3} P-4^{3} S\right)$ in a dense plasma are investigated. Based on a quantum statistical theory the electronic contributions to the shift and width are considered, using the method of thermodynamic Green functions. Dynamic screening of the electron-atom interaction is included. Compared to the width, the electronic shift is more affected by dynamical screening. This effect is increasing at high density. A cut-off procedure for strong collisions is used. The contribution of the ions is taken into account in a quasi-static approximation, with both the quadratic Stark effect and the quadrupole interaction included. The results for shift and width agree well with the available experimental and theoretical data.
\end{abstract}

Keywords: Line shape, Stark broadening, plasma diagnostics, helium spectral line shapes PACS number(s): 52.70.-m,32.70.Jz

\section{INTRODUCTION}

Line profile calculations are an interesting tool for both laboratory and astrophysical plasmas, e.g. to determine the internal parameters or to understand the microscopic processes within the plasma $[1,2]$. Helium spectral lines can be used for diagnostics of laboratory plasmas such as shock wave tube or pulsed arc plasmas [3-6], and in the astrophysical context, of stellar atmospheres of hot stars and white dwarfs [7-10]. The $7065 \AA$ line is used in radiative transfer calculations for supernovae $[11,12]$ and cataclysmic variables [13]. The $6678 \AA$ line is of importance to determine physical properties of massive compact binaries [14]. The line $4713 \AA$ is a dominant one in helium-rich hot sub-dwarfs [15]. The line $7281 \AA$ is observed in P Cyg [16]. The two lines $5048 \AA$ and $4713 \AA$ appear in the spectrum of CI Cam ( $\mathrm{sgB}[\mathrm{e}]$ star), and they are less optically thick than most of the helium lines in the spectrum [17]. Furthermore, He-like spectral lines are a prominent feature in laser-generated plasmas [18-22]. In addition to this, a detailed analysis of the broadening and shift of helium spectral lines of dense plasmas can be used to test our understanding of correlation effects in these plasmas [23, 24].

A review of recent experimental data for Stark broadening of nonhydrogenic neutral atoms is given by Konjević et al. [25, 26]. In particular, Refs. [3-7, 21, 22, 27-32] concern the lines studied in this paper. Stark broadening of several isolated neutral helium lines such as $7281 \AA, 7056 \AA, 6678 \AA, 5016 \AA, 4713 \AA$ and $3889 \AA$ emitted from dense plasmas have been measured in a low pressure pulsed arc plasma by Pérez et al. [3-5]. In these experiments, the electron density was determined by interferometry for different wavelengths, and the plasma electron temperature was estimated from a Boltzmann plot or the intensity ratio of the ion and neutral lines. Stark shift of the He I lines $3889 \AA, 5016 \AA, 7281 \AA$ and $6678 \AA$ have been measured by Djeniže et al. [28] in a linear pulsed arc plasma, using a shot by shot technique. Further experiments are reported for the He I in Refs. [18, 33-46] such as the Stark profile measurement of the He I at $5876 \AA$ performed by Büscher et al. [34] for an electron density in the range of $N_{e}=(0.5-2.5) \times 10^{18} \mathrm{~m}^{-3}$ and a temperature of $T=(4-5.5) \mathrm{eV}$. Deviation from a linear density dependency of Stark broadening parameters has been observed. Profiles of the He I $4471 \AA$ and $4922 \AA$ spectral lines were measured by Frank et al. [35]. The relatively weak forbidden components appear on the wing of the corresponding allowed transitions. Both mentioned transitions are investigated by Adler and Piel [36] at low electron densities ranging from $N_{e}=\left(10^{20}-10^{21}\right) \mathrm{m}^{-3}$.

To calculate the line profile in dense plasmas on a microscopic level, modifications due to the surrounding particles must be taken into account. Screening as an important collective effect in a plasma has to be considered. Note that the influence of electrons and ions can be treated separately due to the difference in mass and mobility. Various approaches have been modified to account these effects [47-52]. Helium series were calculated from semiclassical approach by Griem et al. [51, 52], using a traditional impact approximation for electrons with cut-off procedure, while almost stationary heavy ions are treated in a quasi-static ion approximation due to static microfield.

At low electron densities, moving ions cause a considerable change in the value and the direction of the electric field. 
This leads to additional broadening of spectral lines which is not taken into account by a static ion microfield [53]. Note also that the dynamical treatment of ions is important for overlapping lines. The influence of the motion of the perturbing ions on the Stark line shapes can be considered, e.g., within the model microfield method (MMM) [54] or by molecular dynamics simulations of the line profiles [23]. Molecular dynamics simulations have been performed by Gigosos et al. [23, 55] by introducing two kinds of simulations for calculating He I Stark line profiles. At relatively high electric fields mixing of different sublevels for transitions involving highly excited states leads to forbidden transitions and increasing importance of the linear Stark effect [8].

Recently, a quantum statistical many body approach taking into account medium effects by using thermodynamic Green function technique has been developed [56, 57]. In principle this approach is able to describe dynamical screening and strong collisions by electrons, as well as the dynamic ion microfield in a systematic way. In contrast to molecular dynamics simulations, a consistent quantum description is performed. It has been successfully applied to Lyman and Balmer lines of dense hydrogen plasmas [58-60]. In this work, the Green function approach to spectral line shapes of non-ideal plasmas is extended to helium lines. A review of the basic formalism is presented in Sec. 2 . In Sec. 3 , the shift and full width at half maximum (FWHM) for isolated, non-overlapping He I lines $7281 \AA$, $7065 \AA$, $6678 \AA, 5048 \AA, 4713 \AA$ and electronic contributions of line $4922 \AA$ are evaluated and discussed. Results for the shift and broadening are compared with a number of recent experiments and theoretical calculations. Finally, conclusions are given in Sec. 4.

\section{THEORY OF SPECTRAL LINES IN DENSE PLASMAS}

A quantum statistical approach has been developed to account in a systematic way for medium modifications of spectral line shapes $[56,61,62]$. It starts from the relation of the absorption coefficient $\alpha(\omega)$ and the refraction index $n(\omega)$ to the dielectric function $\epsilon(\mathbf{q}, \omega)$ in the long wavelength limit $q \rightarrow 0$,

$$
\begin{aligned}
& \alpha(\omega)=\frac{\omega}{c n(\omega)} \lim _{q \rightarrow 0} \operatorname{Im} \varepsilon(\mathbf{q}, \omega) \\
& n(\omega)=\frac{1}{\sqrt{2}} \lim _{q \rightarrow 0}\left\{\operatorname{Re} \varepsilon(\mathbf{q}, \omega)+\left[(\operatorname{Re} \varepsilon(\mathbf{q}, \omega))^{2}+(\operatorname{Im} \varepsilon(\mathbf{q}, \omega))^{2}\right]^{1 / 2}\right\}^{1 / 2} .
\end{aligned}
$$

The dielectric function can be determined from the polarization function $\Pi(\mathbf{q}, \omega)$ according to $\epsilon(\mathbf{q}, \omega)=$ $1-V(q) \Pi(\mathbf{q}, \omega)$, where $V(q)=e^{2} /\left(\epsilon_{0} \Omega_{0} q^{2}\right)$ is the Fourier transformed Coulomb potential. Using thermodynamic Green functions, a systematic perturbative treatment of the polarization function can be performed.

The polarization function $\Pi(\mathbf{q}, \omega)$ is related to the dipole-dipole autocorrelation function [57]. The following expression for the full profile $I(\omega)$ is obtained as a convolution of the Doppler-broadened line profile with the pressurebroadened line profile $I^{\mathrm{pr}}(\omega)$ :

$$
I(\omega) \sim \int_{-\infty}^{\infty} \frac{d \omega^{\prime}}{\omega^{\prime}} \exp \left[-\frac{m_{i} c^{2}}{2 k_{\mathrm{B}} T}\left(\frac{\omega-\omega^{\prime}}{\omega^{\prime}}\right)^{2}\right] I^{\mathrm{pr}}\left(\omega^{\prime}\right)
$$

where $m_{i}$ is the mass of the radiating atom and $c$ is the speed of light. The perturber-radiator interaction leads to a pressure broadening (Stark broadening), which contains an electronic and an ionic contribution. Describing the ionic contribution in the quasi-static approximation by averaging over the ionic microfield [47, 57, 63], we get

$$
I^{\mathrm{pr}}(\omega) \sim \sum_{i, i^{\prime}, f, f^{\prime}} I_{i, i^{\prime}}^{f, f^{\prime}}(\omega) \int_{0}^{\infty} d \beta P(\beta) \operatorname{Im}\left\langle i\left|\left\langle f\left|\left[\hbar \omega-\hbar \omega_{i f}-\Sigma_{i f}(\omega, \beta)+\mathrm{i} \Gamma_{i f}^{\mathrm{V}}\right]^{-1}\right| f^{\prime}\right\rangle\right| i^{\prime}\right\rangle .
$$

Here, $\hbar \omega_{i f}=E_{i}-E_{f}$ is the unperturbed transition frequency between the initial $i$ and the final $f$ states, $i^{\prime}$ and $f^{\prime}$ are the corresponding intermediate states

$$
I_{i, i^{\prime}}^{f, f^{\prime}}(\omega)=\langle i|\mathbf{r}| f\rangle\left\langle f^{\prime}|\mathbf{r}| i^{\prime}\right\rangle \frac{\omega^{4}}{8 \pi^{3} c^{3}} \mathrm{e}^{-\frac{\hbar \omega}{k_{\mathrm{B}} T}}
$$

where $\langle i|\mathbf{r}| f\rangle$ has to identified as a dipole matrix element for the transition between $i$ and $f$ states. The ionic microfield $P(\beta)$ is taken according to the Hooper microfield distribution and $\beta=E / E_{0}$ is the normalized microfield strength at ion density $N_{i}=N_{e}$ with Holtsmark normal field strength $E_{0}=e /\left(4 \pi \epsilon_{0} r_{0}^{2}\right)$ where $\frac{4}{15}(2 \pi)^{3 / 2} r_{0}^{3} N_{i}=1[64]$. The line 
profile itself is determined by the vertex correction $\Gamma_{i f}^{V}$ for the overlapping lines and the self-energy corrections of the initial $\Sigma_{i}$ and final states $\Sigma_{f}$

$$
\Sigma_{i f}(\omega, \beta)=\operatorname{Re}\left[\Sigma_{i}(\omega, \beta)-\Sigma_{f}(\omega, \beta)\right]+\mathrm{i} \operatorname{Im}\left[\Sigma_{i}(\omega, \beta)+\Sigma_{f}(\omega, \beta)\right] .
$$

Electronic as well as ionic contributions occur in the self-energy $\Sigma_{n}(\omega, \beta)$, which is assumed to be diagonal in the atomic state $n$,

$$
\Sigma_{n}(\omega, \beta)=\Sigma_{n}^{\mathrm{ion}}(\beta)+\Sigma_{n}^{\mathrm{el}}(\omega, \beta) .
$$

We describe expressions to calculate the self-energy and vertex contributions. Performing a Born approximation with respect to the perturber-radiator interaction, the electronic self-energy is obtained as [57]

$$
\begin{aligned}
\Delta_{n}^{\mathrm{SE}}+\mathrm{i} \Gamma_{n}^{\mathrm{SE}}= & <n\left|\Sigma^{\mathrm{el}}\left(E_{n}, \beta\right)\right| n>=-\frac{1}{e^{2}} \int \frac{\mathrm{d}^{3} q}{(2 \pi)^{3}} V(q) \sum_{\alpha}\left|M_{n \alpha}(\mathbf{q})\right|^{2} \\
& \times \int_{-\infty}^{\infty} \frac{\mathrm{d} \omega}{\pi}\left[1+n_{\mathrm{B}}(\omega)\right] \frac{\operatorname{Im} \varepsilon^{-1}(\mathbf{q}, \omega+\mathrm{i} 0)}{E_{n}-E_{\alpha}(\beta)-(\omega+\mathrm{i} 0)} .
\end{aligned}
$$

Here, the level splitting due to the ion microfield has been neglected [65], $n_{\mathrm{B}}(\omega)=\left(\exp \left(\hbar \omega / k_{\mathrm{B}} T\right)-1\right)^{-1}$ is the Bose distribution function and $M_{n \alpha}(\mathbf{q})$ are the transition matrix elements given below. The sum over $\alpha$ runs from $n-1$ to $n+1$ discrete bound states for virtual transitions. The inverse dielectric function $\varepsilon^{-1}(\mathbf{q}, \omega)$ contains many particle effects which account for the dynamical screening of the interaction in the plasma,

$$
\operatorname{Im} \varepsilon^{-1}(\mathbf{q}, \omega)=-\frac{\operatorname{Im} \varepsilon(\mathbf{q}, \omega)}{[\operatorname{Re} \varepsilon(\mathbf{q}, \omega)]^{2}+[\operatorname{Im} \varepsilon(\mathbf{q}, \omega)]^{2}} .
$$

The random phase approximation (RPA) for the dielectric function is used

$$
\varepsilon^{\mathrm{RPA}}(\mathbf{q}, \omega)=1-2 V(q) \int \frac{d^{3} p}{(2 \pi)^{3}} \frac{f_{e}\left(E_{\mathbf{p}}\right)-f_{e}\left(E_{\mathbf{p}+\mathbf{q}}\right)}{E_{\mathbf{p}}-E_{\mathbf{p}+\mathbf{q}}-\hbar(\omega+\mathrm{i} 0)},
$$

where $E_{\mathbf{p}}=\hbar^{2} \mathbf{p}^{2} / 2 m_{e}$ is the kinetic energy of electrons and $f_{e}\left(E_{\mathbf{p}}\right)$ is the Fermi distribution function of the electrons

$$
f_{e}\left(E_{\mathbf{p}}\right) \simeq \frac{1}{2} N_{e}\left(\frac{2 \pi \hbar^{2}}{m_{e} k_{\mathrm{B}} T}\right)^{3 / 2} \exp \left(-\frac{\hbar^{2} p^{2}}{2 m_{e} k_{\mathrm{B}} T}\right) .
$$

The full expression of the inverse dielectric function has to be used if the transition frequency $\omega_{n \alpha}$ becomes comparable to the electron plasma frequency $\omega_{\mathrm{pl}}=\left(N_{e} e^{2} / \epsilon_{0} m_{e}\right)^{1 / 2}$. However, in the high frequency limit $\omega_{n \alpha}>>\omega_{\mathrm{pl}}$, the inverse dielectric function can be approximated by

$$
\operatorname{Im} \varepsilon^{-1}(\mathbf{q}, \omega) \approx-\operatorname{Im} \varepsilon(\mathbf{q}, \omega)
$$

This binary collision approximation leads to a linear behavior of the electronic shift contribution with respect to the electron density, whereas a nonlinear dependence of the electronic shift with increasing electron density is expected if the full expression of the inverse dielectric function is used [65, 66]. For virtual transitions between states with the negligible energy difference, the static Debye screening can be considered for the inverse dielectric function such as $[58,65]$ :

$$
\operatorname{Im} \varepsilon^{-1}(\mathbf{q}, 0)=\frac{\operatorname{Im} \varepsilon(\mathbf{q}, 0)}{\left(1+\frac{\kappa^{2}}{q^{2}}\right)^{2}}
$$

where $\kappa=r_{\mathrm{D}}^{-1}=\left[2 e^{2} N_{e} /\left(\epsilon_{0} k_{\mathrm{B}} T\right)\right]^{1 / 2}$ is the inverse Debye radius. On the other hand, the static Debye screening can be also applied in the semi-classical calculation by using the cut-off parameter $\rho_{\max }=1.1 r_{\mathrm{D}}$, according to Griem [52]. In our case the lower limit $q_{\min }=1 / \rho_{\max }$ of the $q$-integration can be selected.

The vertex function for the coupling between the upper and the lower state is given by

$$
\Gamma_{i f}^{V}=\frac{2 \pi}{e^{2}} \int \frac{d^{3} q}{(2 \pi)^{3}} \frac{d^{3} p}{(2 \pi)^{3}} f_{e}\left(E_{\mathbf{p}}\right) V^{2}(q) M_{i i}(\mathbf{q}) M_{f f}(-\mathbf{q}) \delta\left(\frac{\hbar^{2} \mathbf{p} \cdot \mathbf{q}}{m_{e}}\right) .
$$


The transition matrix elements $M_{n \alpha}(\mathbf{q})$ describe the coupling between free charges and bound states. In lowest order, they are determined by the atomic eigenfunctions $\psi_{n}(\mathbf{P})$ of the radiating electron and depend on the momentum transfer $\hbar \mathbf{q}[56,61]$,

$$
\begin{aligned}
M_{n \alpha}(\mathbf{q}) & =\int \frac{\mathrm{d}^{3} p}{(2 \pi)^{3}} \psi_{n}^{*}(\mathbf{p})\left[Z_{n} e \psi_{\alpha}(\mathbf{p})-e \psi_{\alpha}(\mathbf{p}+\mathbf{q})\right] \\
& =\mathrm{i} e\left(Z_{n} \delta_{n \alpha}-\int \mathrm{d}^{3} r \psi_{n}^{*}(\mathbf{r}) \exp (\mathrm{iq} \cdot \mathbf{r}) \psi_{\alpha}(\mathbf{r})\right)
\end{aligned}
$$

assuming $m_{e} \ll m_{i}$ and $Z_{n}$ is the ion charge. Expanding the plane wave into spherical harmonics

$$
\exp (\mathrm{iq} \cdot \mathbf{r})=4 \pi \sum_{l=0}^{\infty} \sum_{m=-l}^{l} \mathrm{i}^{l} j_{l}(q r) Y_{l m}^{*}\left(\Omega_{q}\right) Y_{l m}\left(\Omega_{r}\right)
$$

a multipole expansion can be derived, e.g. $l=0,1,2$ gives the monopole, dipole and quadrupole contribution of the radiator-electron interaction, respectively.

Contrary to hydrogen or hydrogen-like ions, the bound state wave functions are not explicitly known for helium. Approximations have to be made. Here, we assume for the spherical part of the wave function a linear combination of hydrogen-like wave functions, while the radial part of the matrix element in dipole transition is calculated based on Coulomb approximation method of Bates and Damgaard [67-69]. In more detail, $P_{n, l}(r)$ is related to the normalized radial eigenfunctions of the jumping electron. A series expansion of these wave functions in terms of hydrogenic wave functions is considered:

$$
P_{n l}(r)=r R_{n l}(r)=\left(\frac{2 r}{n^{*}}\right)^{n^{*}} \exp \left(-\frac{r}{n^{*}}\right) \sum_{k=0}^{k_{\max }} \frac{a_{k}}{r^{k}} .
$$

The effective principal quantum number $n^{*}$ depends on the ionization energy of neutral helium and the energy of the corresponding state according to

$$
\begin{aligned}
n^{*} & =\sqrt{\frac{1 \mathrm{Ry}}{E_{\infty}-E_{n l}}}, \\
a_{k} & =a_{k-1} \frac{n^{*}\left[l(l+1)-\left(n^{*}-k\right)\left(n^{*}-k+1\right)\right]}{2 k}, \\
a_{0} & =1 / n^{*}\left[\Gamma\left(n^{*}+l+1\right) \Gamma\left(n^{*}-l\right)\right]^{1 / 2} .
\end{aligned}
$$

To ensure convergence, the series should be truncated [68] by $a_{k}=0$ for $k_{\max }=k>n^{*}+0.5$.

As stated above, the electronic contribution of the self-energy is evaluated in the Born approximation. This overestimates the self-energy. In particular, strong collisions with large momentum transfer are not treated appropriately by the Born approximation, while the perturbation theory breaks down at small distances. A systematic calculation for strong collisions is possible by partial summation of diagrams forming a three-particle T-matrix [57] or by using the close-coupling method [70]. To avoid a perturbative expansion with respect to the interaction, strong collision contributions to the broadening are estimated by a Lorentz-Weisskopf approximation [51, 52, 69] with the introduction of a cut-off parameter for the $q$-integration see Eq. (8). Following Griem, this cut-off parameter is the inverse of the minimum limiting impact parameter $\left(q_{\max }=1 / \rho_{\min }\right)[57]$

$$
\begin{aligned}
\rho_{\min } & =\left[\frac{2 \mathrm{e}^{4}}{3 \hbar^{2} v^{2}} \sum_{\alpha}|<i| \mathbf{r}|\alpha>|^{2}\left|A\left(z_{i \alpha}^{\min }\right)+i B\left(z_{i \alpha}^{\min }\right)\right|\right]^{1 / 2}, \\
z_{i \alpha}^{\min } & =\left(E_{i}-E_{\alpha}\right) \rho_{\min } / \hbar v \\
A(z) & =z^{2}\left[K_{0}^{2}(|z|)+K_{1}^{2}(|z|)\right] \\
B(z) & =\pi z^{2}\left[K_{0}(z) I_{0}(z)-K_{1}(z) I_{1}(z)\right]
\end{aligned}
$$

where $I$ and $K$ are modified Bessel functions [52]. An appropriate strong electronic collision term for the width of neutral helium, which is also introduced by Griem et al. [51], should be added to the width in Eq. (8), and the shift according to Eq. (3.17) in Ref. [51] as following: 


$$
\Delta^{\text {total }}=\Delta_{n}^{S E}+\left(\frac{4}{3}\right)^{2 / 3} N_{e} \int v \pi \rho_{\min }^{2} f(v) d v,
$$

with the Maxwellian velocity distribution function,

$$
f(v)=\left(\frac{m_{e}}{2 \pi k_{\mathrm{B}} T_{e}}\right)^{3 / 2} \exp \left(-\frac{m_{e} v^{2}}{2 k_{\mathrm{B}} T_{e}}\right),
$$

where $v$ is electron velocity, and $N_{e}$ is the electron density.

For the ionic contribution to the self-energy, we approximate the time-dependent microfield fluctuation by its static value. In general, dynamic ionic microfield is important for overlapping lines and at low electron density in the line center $[71,72]$. Due to the slow movement of heavy ions, the ion microfield is assumed to be constant during the time of interest for the radiation process. The perturbing ions can be considered to be static during the radiation except in the line center. The static ionic contribution to the ionic self-energy is treated by means of the microfield concept including both quadratic Stark effect and quadrupole effects. The first order perturbation term vanishes for non-hydrogenic like atoms because of nondegeneracy with respect to the orbital quantum number $l$. According to second order perturbation theory, the quadratic Stark effect is proportional to the square of the microfield [73]:

$$
\Sigma_{n l m}^{2}(E)=e^{2}|E|^{2} \sum_{n^{\prime}, l^{\prime}, m^{\prime}} \frac{|<n, l, m| z\left|n^{\prime}, l^{\prime}, m^{\prime}>\right|^{2}}{E_{n l m}-E_{n^{\prime} l^{\prime} m^{\prime}}},
$$

where $E$ is the microfield strength, $n, l$, and $m$ are the well known principal, orbital, and magnetic atomic quantum numbers, respectively. The quadrupole Stark effect is due to the inhomogeneity of the ionic microfield. We use the expression derived by Halenka [74]

$$
\Sigma_{n n^{\prime}}^{3}(E)=-\frac{5}{2 \sqrt{32 \pi}} \frac{e E_{0}}{r_{0}} B_{\rho}(\beta)<n\left|3 z^{2}-r^{2}\right| n^{\prime}>
$$

Here, $B_{\rho}(\beta)$ is the mean field gradient at a given field strength and the screening parameter $\rho=r_{0} / r_{\mathrm{D}}$ is taken as the ratio between the mean particles distance $r_{0}$ (see below Eq. 5) and the Debye radius $r_{\mathrm{D}}$.

\section{RESULTS}

Applying the formalism outlined above, helium line profiles can be calculated for given electron densities and temperatures. From these profiles, a full width at half maximum (FWHM) and a shift of the peak position can be determined; the value of the line shift is obtained from the distance between the unperturbed line and the peak position of the profile, while the full width of the profile is determined from the positions whose intensity are half the intensity of the maximum of the line. Note that different expressions of shift, like shift of center of mass or shift at half-width exist [30]. Here, our definition follows the definitions used in the experimental studies.

We present results of the spectral line shapes of the isolated He I lines $\left(2^{1} P-3{ }^{1} S\right),\left(2^{3} P-3^{3} S\right),\left(2^{1} P-3^{1} D\right)$, $\left(2^{1} P-4^{1} S\right)$, and $\left(2^{3} P-4^{3} S\right)$. Also the electronic shift and width for the transition $\left(2^{1} P-4^{1} D\right)$ in dense plasmas is calculated. However, the profile of this line merges with it's forbidden component [35, 36]. The Born approximation with respect to the dynamically screened electron-atom interaction is given by Eq. (9) and binary approximation has been considered, according to Eq. (12). As an example, we compare the spectral profile for the transitions $7281 \AA\left(2^{1} P-3^{1} S\right)$ and $6678 \AA\left(2^{1} P-3^{1} D\right)$ in Figs. 1 and 2 with simulation results obtained by Gigosos et al. [75]. As can be seen, a perfect agreement between our results in binary approximation and the simulation data is found for the $7281 \AA$ in the line center, small discrepancies arise in the line wings, while in the case of dynamical screening the line shift becomes smaller. As for the $6678 \AA$ line, the discrepancy is somewhat larger, still the overall agreement is reasonable. The simulation result shown here is used only for independent particles. Comparison with the more realistic case of interacting particles would of course be more meaningful [75]. Note, that the asymmetry of the spectral line shape is small in the case of $7281 \AA$ line but larger for the $6678 \AA$ line. 


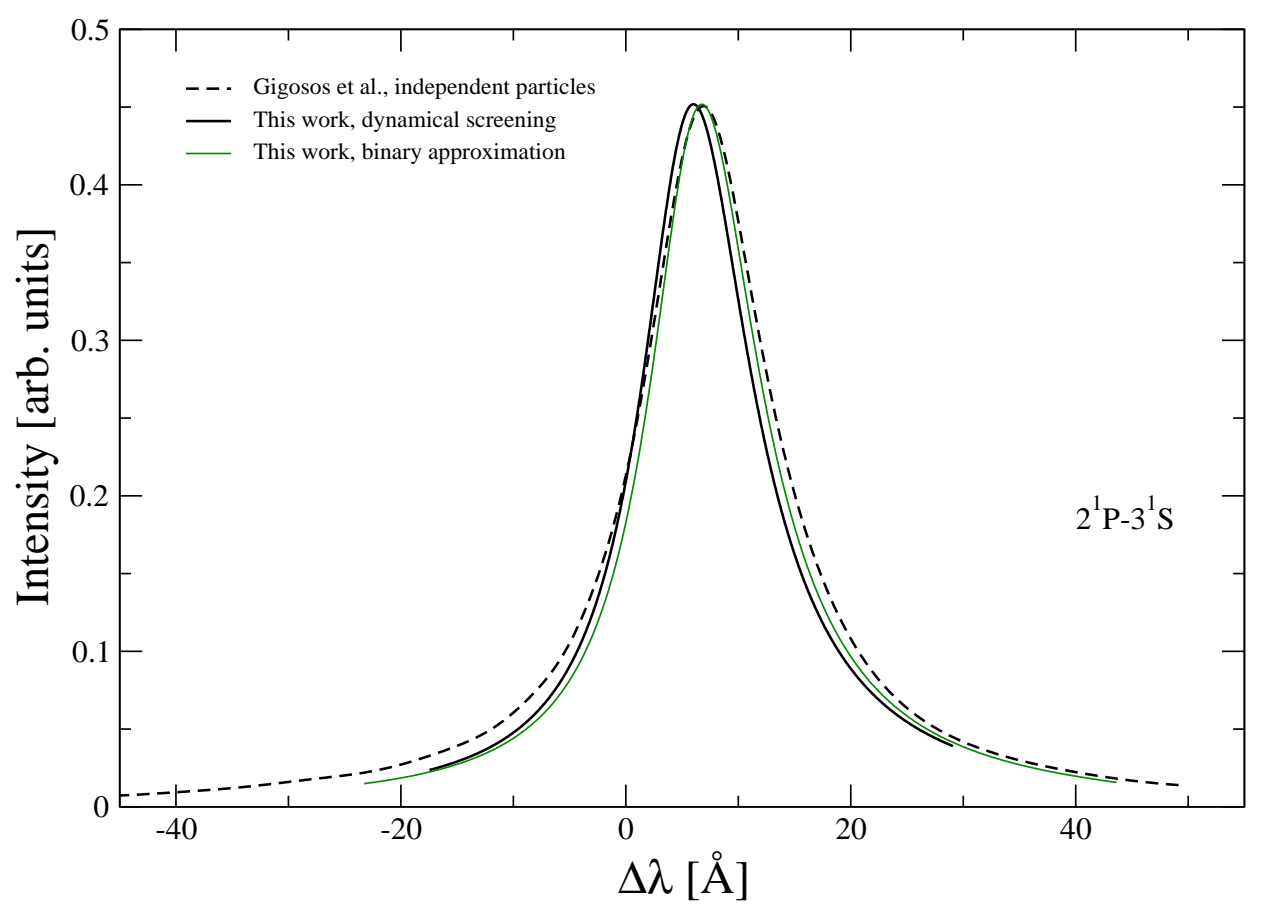

FIG. 1: (color online) Theoretical spectral line profile (solid line) of He I line $7281 \AA$ for an electron density of $1.457 \times 10^{23} \mathrm{~m}^{-3}$ and a temperature of $2 \times 10^{4} \mathrm{~K}$, compared with calculations of Gigosos et al. (broken line) [75].

In Figs. 3 and 4, we show the electronic width (HWHM) and shift for the $4922 \AA$ line, respectively, as a function of temperature at electron density $N_{e}=1.0 \times 10^{22} \mathrm{~m}^{-3}$. Comparison is made with theoretical values given by Griem [52], Bassalo et al. (BCW) [49], and Dimitrijević and Sahal-Bréchot (DSB) [50]. In general our results are of similar size as previous calculations, the temperature dependence is similar as well. The binary approximation result for shift delivers good agreement with the other calculations for this transition. In our calculation the effect of dynamical Debye screening is considered, which leads to reduction of electronic shift and width. In the standard theory of Griem [52] a semiclassical impact approach is used. The importance of the correction for Debye screening at high density was pointed out, especially, when the plasma frequency becomes larger than the splitting between interacting levels [1]. For this transition the reduction of the width is about $20 \%$ in the given temperature range. An approximation for strong electron collisions is attended, in which the perturbation theory breaks down. The cut-off procedure have been developed and used extensively by Griem and collaborations [51, 52, 69], as well as in the semiclassical formalism by Dimitrijević and Sahal-Bréchot [50]. Bassalo et al. [49] assumed in their calculations the convergent method [68] in a many-level approximation.

Next, the total width and the total shift for the line $7281 \AA$ as a function of the electron density are given in Fig. 5 and Fig. 6, respectively. Doppler broadening is included according to Eq. (3). We compare our results with the visible spectrum emitted by a helium plasma generated in a wall-stabilized arc, which is reported by Kelleher [30], the determined electron density was $1.03 \times 10^{22} \mathrm{~m}^{-3}$ at electron and gas temperatures of $2.09 \times 10^{4} \mathrm{~K}$ and $1.58 \times 10^{4}$ $\mathrm{K}$, respectively. The semi-classical approach of Bassalo et al. [76] assumed for Debye screening by introducing the Debye cut-off parameter. The broadening and shift due to electron collisions with and without Debye screening effect were estimated. The ion contribution has been treated in the same way as in the approach developed by Griem [52]. The results obtained by a molecular dynamics (MD) simulation for independent and interacting particles by Gigosos et al. $[23,75]$ cover the density range $(0.25-50.0) \times 10^{22} \mathrm{~m}^{-3}$ and temperature range $(1.9-4.2) \times 10^{4} \mathrm{~K}$, while for the values obtained in a pulse arc plasma by Pérez et al. [4] the temperature lies in the interval $(1.6-2.5) \times 10^{4} \mathrm{~K}$ with a mean value around $2 \times 10^{4} \mathrm{~K}$. They included the estimated theoretical data from Griem approach [52]. In the calculation of Gigosos et al. [75], no Doppler broadening is taken into account.

The overall agreement of our result with results by other authors is quite good. Comparison to the estimated values 


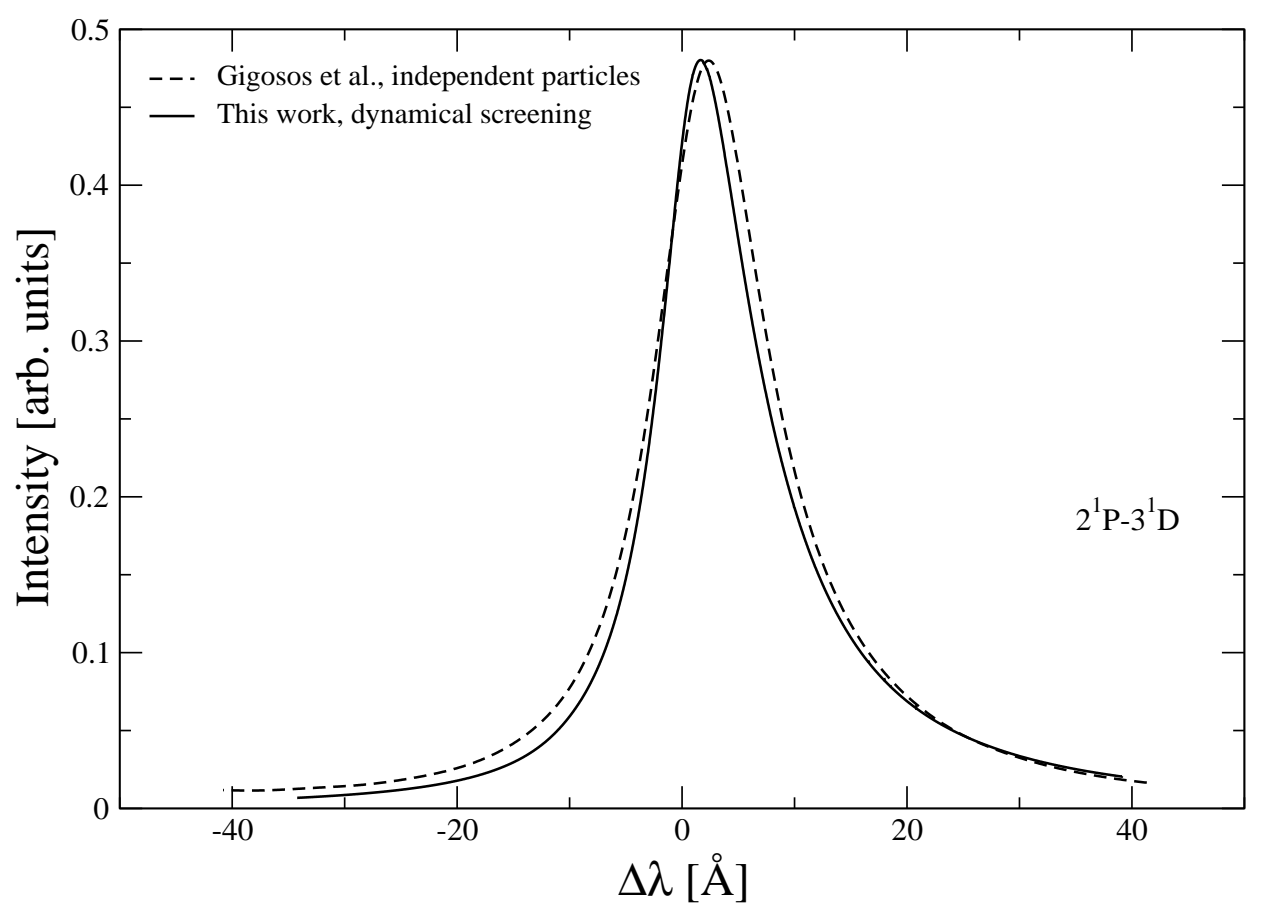

FIG. 2: Theoretical spectral line profile of He I line $6678 \AA$ (solid line) for an electron density of $14.57 \times 10^{22} \mathrm{~m}^{-3}$ and a temperature of $2 \times 10^{4} \mathrm{~K}$, comparison is made with Gigosos et al. [75] (broken line).

of Griem [4, 52], the deviations are more pronounced for the width than for the shift. The discrepancies are larger at very low density. At lower densities the Stark broadening becomes less important, and the relative contribution of Doppler broadening increases. However, ion dynamics which is not considered in this paper becomes important at low densities. This might be the reason for the discrepancies at very low densities.

Our results for shift and FWHM versus electron density for the transition $7065 \AA$ are given in Figs. 7 and 8 , respectively. Comparisons are made with: i) a number of measured [30, 32, 77] and theoretical data [76], ii) observation in a repetitively pulsed low-pressure arc hydrogen-helium plasma measured by Mijatović et al. [27] with an electron density range $(0.25-0.50) \times 10^{22} \mathrm{~m}^{-3}$, electron temperatures of $(1.93-2.36) \times 10^{4} \mathrm{~K}$ and gas temperatures of $(0.5-1.2) \times 10^{4} \mathrm{~K}$, the latter are measured from the Doppler broadening of He I line profiles, iii) theoretical results by Gigosos et al. [23, 75], covering a density range of $(0.25-249) \times 10^{22} \mathrm{~m}^{-3}$ and a temperature range of $(1.93-6.34) \times 10^{4} \mathrm{~K}$, iv $)$ the measured values of Pérez et al. [5] in the plasma density range of $(1-6) \times 10^{22} \mathrm{~m}^{-3}$ and temperature interval of $(0.8-3) \times 10^{4} \mathrm{~K}$ with mean value $2 \times 10^{4} \mathrm{~K}$, v) theoretical values from Griem's approach [52], reported by Pérez et al. [5]. Good agreement between our results and other investigations is found. Again, the width shows some discrepancy at low densities. As before, this might be due to the lack of ion dynamics in our approach. Calculations with Doppler broadening are shown as well.

In order to compare different theoretical approaches, the ratio of the experimental width to the theoretical one is considered. In this comparison the width of the experiment performed by Milosavljević and Djeniže [32] is used as a reference. The measured electron densities and temperatures were in the range $(4.4-8.2) \times 10^{22} \mathrm{~m}^{-3}$ and $(1.8-3.3) \times 10^{4} \mathrm{~K}$ with error bars $\pm 9 \%$ and $\pm 10 \%$, respectively. Fig. 9 shows the ratio of the experimental Stark width to the theoretical scaling data of Griem [52], BCW [49], and DSB [50] as a function of temperature taken from the same Ref. [32]. The ratios between experimental FWHM to various theoretical values show some deviations from unity. Our calculations are also included, lower than Griem's values and agree with the values given according to BCW [49], but they are generally somewhat higher than the measured values.

To assess the importance of different effects, the individual contributions due to electrons and ions are compared in Figs. 10 and 11. As can be seen, the ionic contributions to the line width is about 19\%, agree with the values of Griem, and lower than the values given by Milosavljević and Djeniže [32]. Ionic contributions are significantly smaller than electronic contributions. About $13 \%$ to $21 \%$ of the electronic part is related to the strong collision 


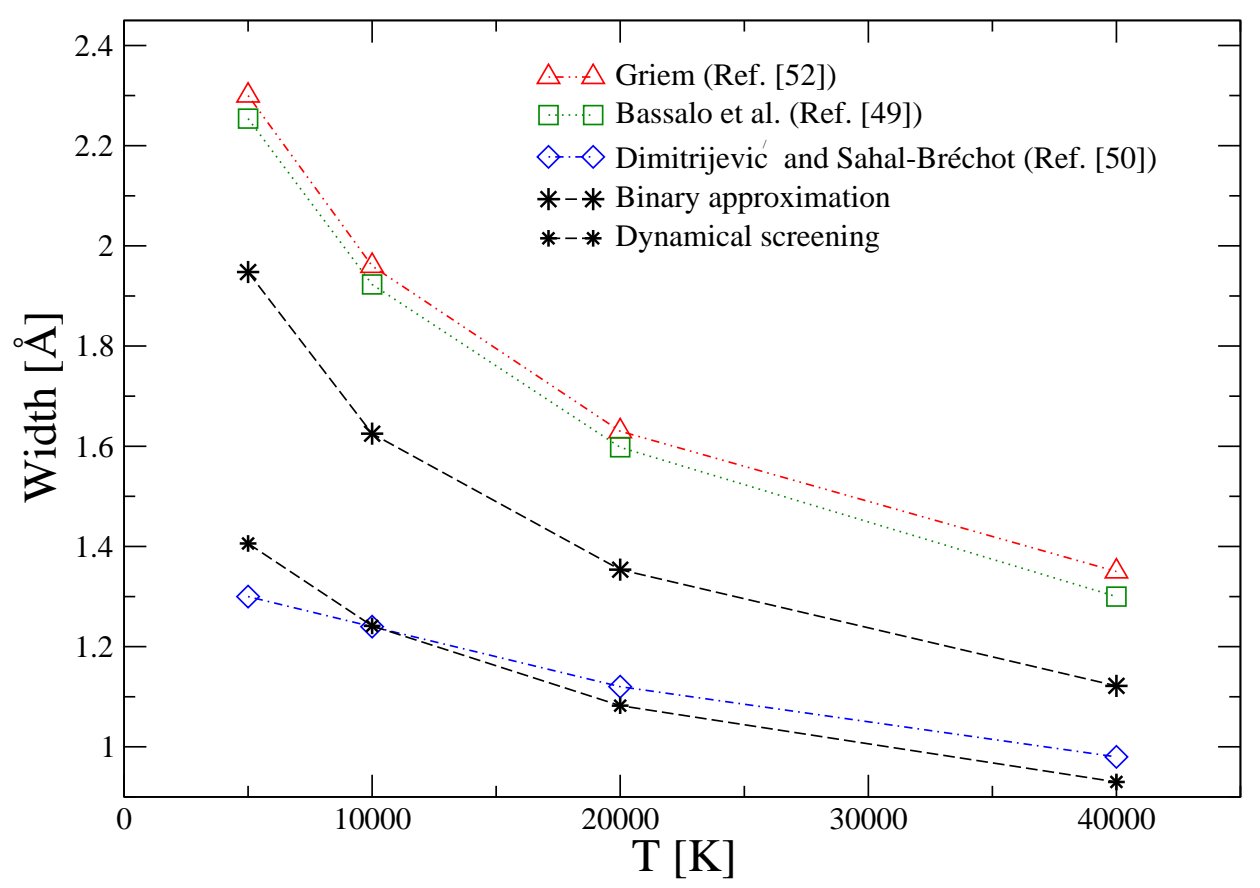

FIG. 3: (color online) Electronic width HWHM of He I line $4922 \AA$ for electron density $1.0 \times 10^{22} \mathrm{~m}^{-3}$ as a function of temperature.

term. Note also, that the estimated values by Milosavljević and Djeniže [32] give slightly higher results for the ion contributions and smaller values for the electron contributions, but the total width is still lower than theoretical values. Furthermore, the measured value of Kelleher [30] is indicated in Figs. 10 and 11.

Similar calculations are performed for the line $6678 \AA$. The comparison is shown for FWHM in Figs. 12,13 and for the shift in Fig. 14. The experimental FWHM data of Vujicić et al. [21] are illustrated in Fig. 12. They have been measured in a laser produced plasma for electron density $N_{e}=(0.7-1.7) \times 10^{23} \mathrm{~m}^{-3}$ at an average temperature of $T=3 \times 10^{4} \mathrm{~K}$, where the validity of the quasi-static approximation is satisfied except in the line center. Also, a forbidden component does not overlap with the isolated one. Comparison was given with the theoretical results of Griem [51, 52], Bassalo et al. [49], Dimitrijević and Sahal-Bréchot [50] by using Griem's approach for the static ion contributions in Ref. [21]. The agreement of the experimental values with our best calculations, i.e. including dynamical screening is better than with the other theoretical approaches. A small discrepancy still remains at high densities, even in case where dynamical screening is included. In Fig. 13, additional data for a larger range of densities are shown: i) values obtained by Pérez et al. [3] for densities from $(2.0-6.46) \times 10^{22} \mathrm{~m}^{-3}$ and temperatures between $(1.9-4.3) \times 10^{4} \mathrm{~K}$, ii) experimental values by Mijatović et al. [27] and Kelleher [30], iii) measurement by Milosavljević and Djeniže [31] was performed at electron densities between $(0.3-8.2) \times 10^{22} \mathrm{~m}^{-3}$ and electron temperatures of $(8-3.3) \times 10^{4} \mathrm{~K}$ in five different plasma discharge conditions using a linear, low-pressure, pulsed arc as an optically thin plasma source, operated in a helium-nitrogen-oxygen gas mixture. Comparison with the theoretical results by Bassalo et al. [76] are given as well. Furthermore, the results by Gigosos et al. [23, 75] in the density range $(0.25-50.0) \times 10^{22} \mathrm{~m}^{-3}$ and temperature range $(1.9-4.2) \times 10^{4} \mathrm{~K}$ are included.

The Doppler broadening become less important with increasing density, and the width is only due to Stark broadening at high densities. In general, our calculated FWHM results for the given densities and temperatures agree with the other theoretical and experimental values. The theoretical scaling data of Griem for broadening was also given by Pérez et al. [3]. We include the line shift in the same manner from Ref. [52]. The estimated values of Griem [52] are systematically higher than our values, especially for the line shift.

As before, the inclusion of dynamical screening reduces the magnitude of both width and shift. It also causes some non-linear behavior at high densities, see Fig. 14. We find that the shift of this line is more sensitive to plasma screening, as the virtual transitions to neighboured states do not contribute to the line profile if the energy difference is comparable to the plasma frequency $[66,78]$. The discrepancy between our results and the available data is more 


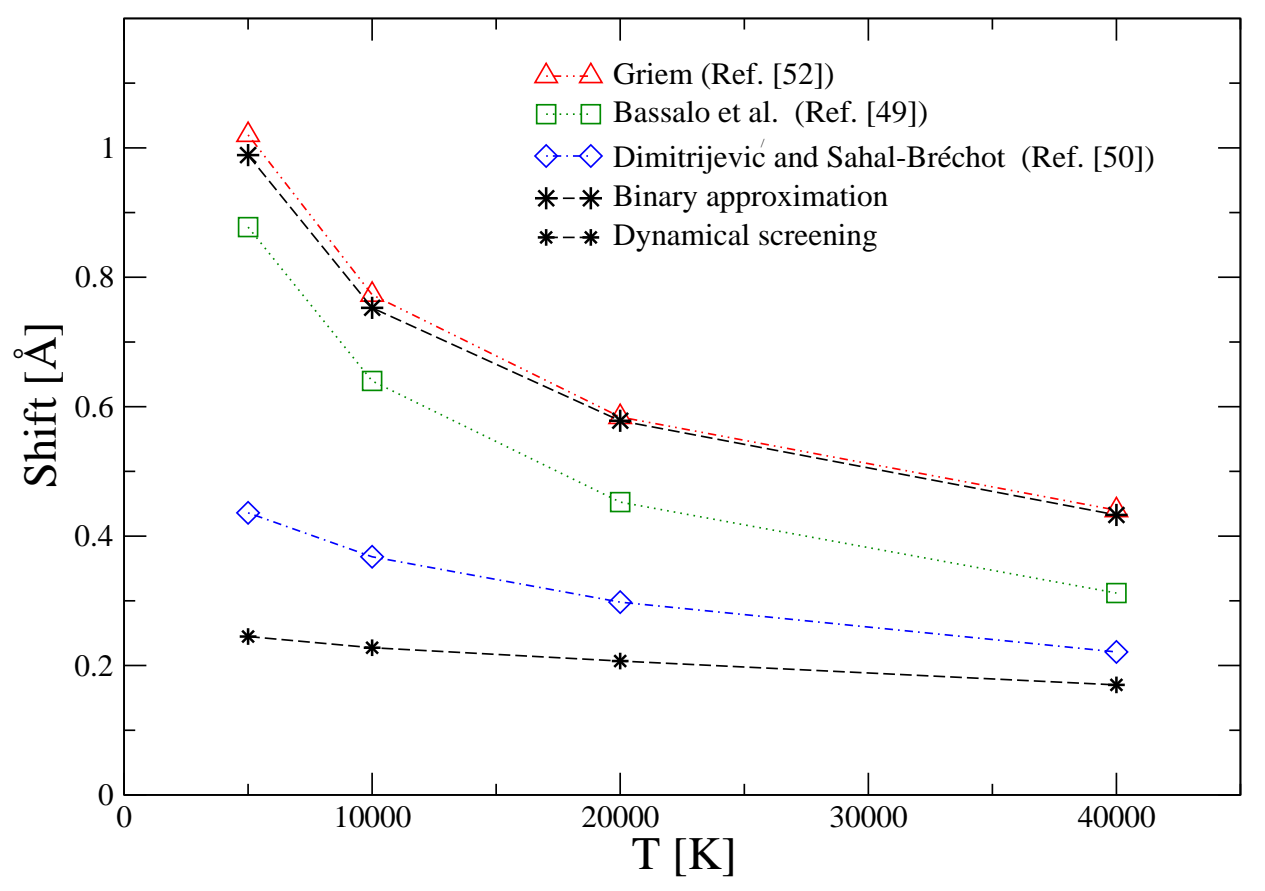

FIG. 4: (color online) Electronic shift of He I line $4922 \AA$ for electron density $1.0 \times 10^{22} \mathrm{~m}^{-3}$ as a function of the temperature.

\begin{tabular}{|cccccccc|}
\hline$N_{\mathrm{e}}\left(10^{22} \mathrm{~m}^{-3}\right)$ & $T_{e}\left(10^{3} \mathrm{~K}\right)$ & $W_{\mathrm{B}}(\AA)$ & $W_{\mathrm{th}}(\AA)$ & $W_{\exp }(\AA)$ & $d_{\mathrm{B}}(\AA)$ & $d_{\mathrm{th}}(\AA)$ & $d_{\exp }(\AA)$ \\
\hline 2.0 & 18.0 & $3.10 / 3.10$ & $3.183 / 3.179$ & 3.4 & $1.43 / 1.35$ & $1.783 / 1.605$ & $0.9[80]$ \\
1.03 & 20.9 & $1.58 / 1.58$ & $1.623 / 1.622$ & 1.68 & $0.75 / 0.68$ & $0.862 / 0.788$ & $0.89[30]$ \\
\hline
\end{tabular}

TABLE I: Comparison of the calculated (values without/with screening are given) and experimental results for FWHM and shift are given for line $5048 \AA ; W_{\text {th }}$ and $d_{\text {th }}$ this work; $W_{\mathrm{B}}$ and $d_{\mathrm{B}}$, Bassalo et al. [76]. The experimental values $W_{\exp }$ and $d_{\exp }$ have been taken from Table I and II given by Bassalo et al. [76].

pronounced for the shift. For low densities $N_{e}<10^{22} \mathrm{~m}^{-3}$, the calculated shift is about half the size of the MD data of Gigosos et al. [75], while at high densities we overestimate the shift in binary approximation as compared to the MD results. Concerning the high densities, the data by Gigosos et al. [75] already indicate the importance of strong coupling effects. Regarding the data point reported by Bassalo et al. [76], the discrepancy might be due to neglect of the shift and width in the lower energy states. Bassalo et al. [76] use the Debye radius for the maximum impact parameter and take the reduced matrix elements from the oscillator strength of the transition. In particular for the line shift, the discrepancies might be related to the somewhat ambiguous definition of the cut-off parameter. In addition, the line might be no longer isolated, or the linear Stark effect might be of importance at high electron densities. Note, that an asymmetric profile of this line was already shown in our previous work [79].

The Stark broadening parameters for the transition $2^{1} P-4^{1} S(5048 \AA)$ are given in Tab. I. Our data $W_{\text {th }}$ and $d_{\text {th }}$ are again compared to calculated values of Bassalo et al. [76] and measurements [30, 80]. Good agreement is found.

Stark broadening calculations have been also performed for the line $4713 \AA$. Once again, a linear dependence of the FWHM with the electron density is found in Fig. 15. For this line, Pérez et al. [3] have carried out measurements in the temperature range $(1.9-4.3) \times 10^{4} \mathrm{~K}$. In this case, only results for the width with the corresponding theoretical values from Griem approach [3, 52] have been reported. Besides the experimental results [27, 29, 30, 80], the theoretical values $[29,76]$ for both width and shift are also shown in Figs. 15 and 16. We notice a good agreement between our results and the other data, especially at lower densities. At the highest density some disagreement is found among the various data. However, at this density also the experimental error bars are larger.

For the same transition, spectral line profiles have been measured by Milosavljević and Djeniže [31] in the 


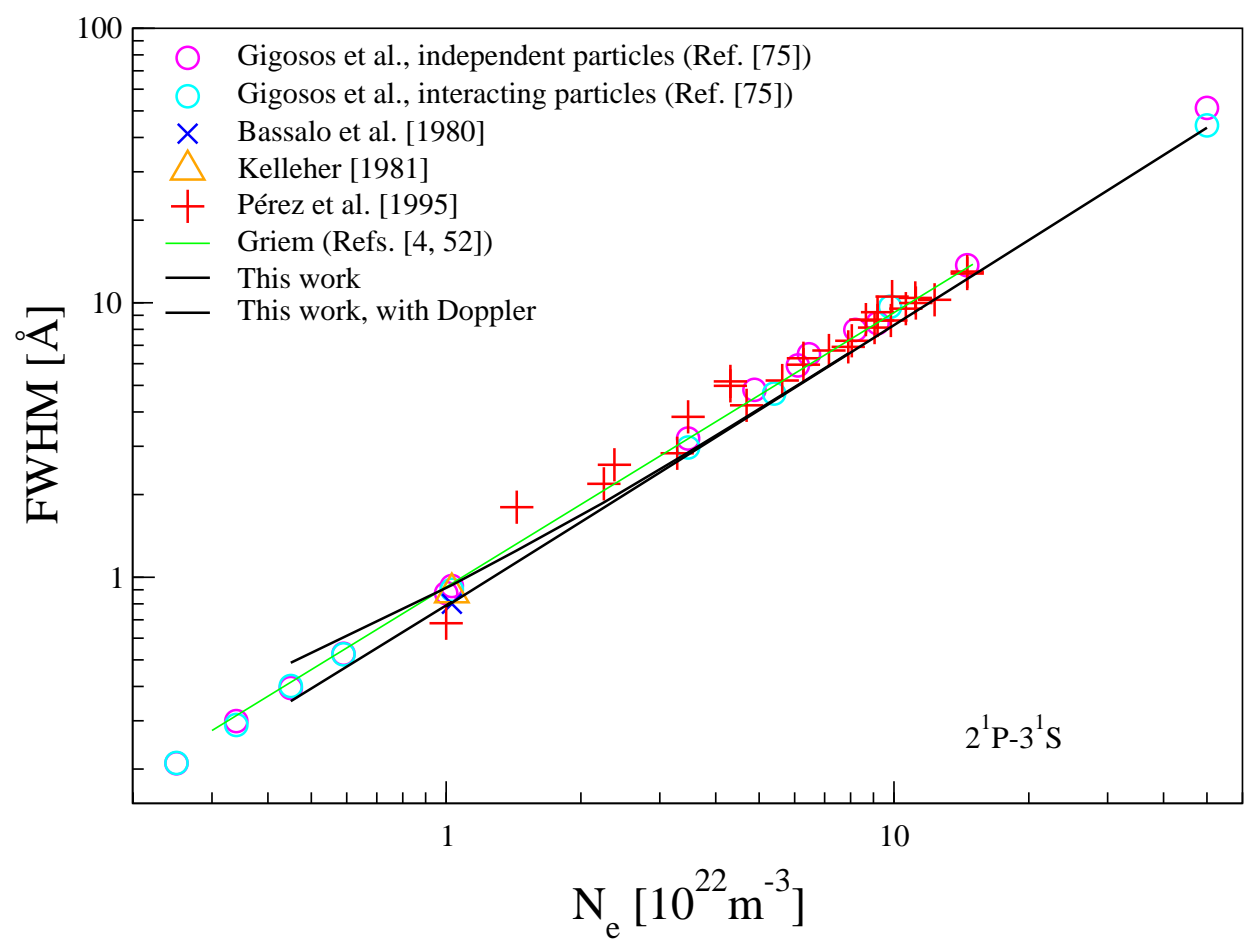

FIG. 5: (color online) The full width at half maximum for the He I line $7281 \AA$ versus electron density. Comparison is made with available theoretical and experimental data. The electron temperature is taken according to references $[4,30,75,76]$, see also Tab. III.

temperature range of $(18-33) \times 10^{3} \mathrm{~K}$ and electron density between $(4.4-8.2) \times 10^{22} \mathrm{~m}^{-3}$. From the observed profiles, using a deconvolution procedure, ion static and dynamic broadening parameters were estimated. However, in this case, dynamical ion contributions play no role [31]. The comparison of our results, the experimental data, the theoretical values of Griem [51, 52], BCW [49], and DSB [81] reported by the same Ref. [31] are presented in Fig. 17. Our electronic contributions lie above BCW [49], the total width is slightly larger than the experimental values but smaller than Griem's approach. Moreover the experimental result of Kelleher [30] is implied.

In order to represent the data more clearly, the values for FWHM and shift obtained in our approach are compared with other theoretical approaches as well as experimental data in Tabs. II and III, respectively.

As mentioned, the binary collision approximation can be evaluated starting with Eq. (12). The results for the line shift $d_{1}$ for line $7065 \AA$ is given in Tab. IV. Based on the binary collision approximation, the line shift $d_{2}$ for static Debye screening can be found by using $\kappa$ as the lower limit of the $q$-integration. The contribution of the dynamical screening effect $d_{3}$ is obtained by using the full expression for the imaginary part of the inverse dielectric function as given in Eq. (9). The correction due to the static Debye screening leads to reduction of the shift at large values of $\kappa$, which correspond to high electron density and relative low temperature but it does not influence the width.

In our calculations, the ions have been treated as stationary during the mean time of an electron collision. The static microfield leads to a discrepancy at very low plasma densities where the motion of ions is more important. The static microfield approach can be improved by using the model microfield method [71, 82] or MD simulations $[23,72,75]$. However, for a nonhydrogenic, isolated neutral atomic line, a simple expression can be given, which closely approximatse the ion dynamics contribution. In particular, following the unified adiabatic theory which was independently developed by Griem [52] and by Barnard, Cooper, and Smith (BCS) [83], such an expression can be given in terms of a dynamic ion broadening parameter [27, 30-32, 40]. This parameter is equal to unity for the measured values of lines $4713 \AA$ and $5016 \AA$ in Ref. [31]. Therefore, the effect of ion dynamics is negligible for the given electron density and temperature. 


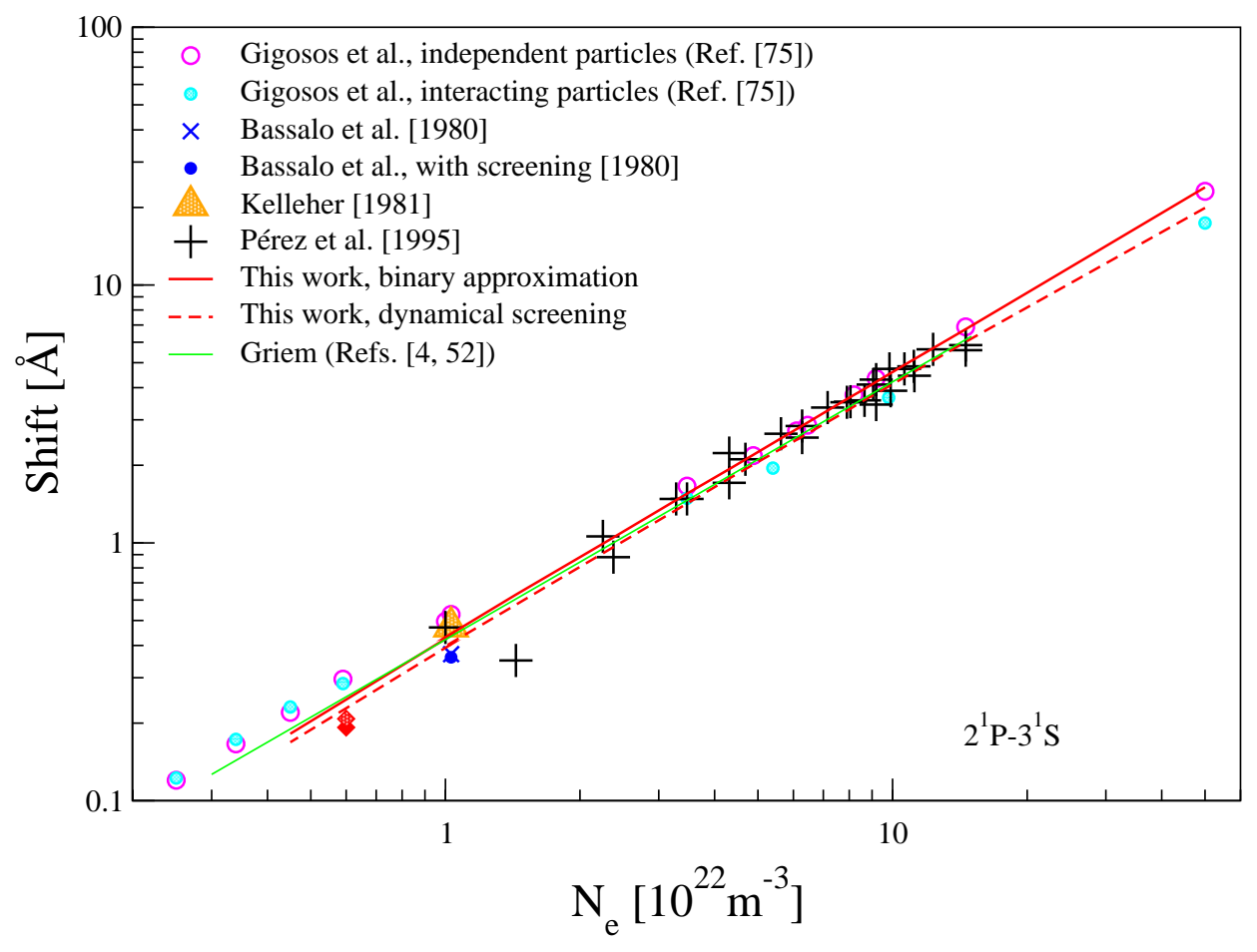

FIG. 6: (color online) The shift of the He I line $7281 \AA$ versus electron density. Comparison is made with available theoretical and experimental data. The electron temperature is taken according to the references $[4,30,75,76]$, see also Tab. II.

\section{CONCLUSIONS}

A quantum statistical approach using the technique of thermodynamic Green function is applied to calculate in-medium modifications of spectral lines in dense plasmas. The approach has been used before to hydrogen and hydrogen-like ionic spectral lines [57,63]. In the present work, this approach has been extended successfully to the helium atom, applied for the electron density range of $\left(10^{16}-10^{18}\right) \mathrm{cm}^{-3}$ and temperature range of $(1-6) \times 10^{4} \mathrm{~K}$.

Recently Stark broadening of several helium lines has been observed in various plasma experiments [5,30,32]. An over all good agreement is found by comparing our results with measured values. For higher densities the behavior of shift and width of spectral lines becomes nonlinear, see also Fig. 12. A similar behavior is already known from hydrogen [58].

Calculations of helium lines were first performed by Griem et al. [51, 52]. Another recent calculations of helium line profiles have been performed by Gigosos et al. [23, 75] based on semiclassical molecular dynamics simulations for independent as well as for interacting particles in non-quenching approximation. In the temperature and density range under consideration both approaches give results generally in good agreement with our calculations, which are strictly quantum statistical and not restricted to a non-quenching approximation. Moreover, in our calculations quadrupole contributions are taken into account. The correction due to this contribution is increasing at high densities. In the region under consideration, all the considered theories give results for the shift and width of spectral lines which are in reasonable agreement. We expect that our quantum statistical approach can also be used for higher densities to obtain relevant results in this region.

In dense plasmas, where the energy distance between the perturbed and the neighboured perturbing energy levels becomes comparable to the electron plasma frequency, dynamical screening effects are important. Screening reduces the shift especially at high electron density in analogy to hydrogen [58]. This behavior is shown in Fig. 6 and Fig. 12. The line width is dominated by the electronic contribution, which is proportional to $N_{e}$. This can be seen in Fig. 11.

At high densities, pair-correlation effects are important for the microfield distribution function. They can be derived by an appropriate approach such as APEX [84, 85] or Monte Carlo simulations [24] in the case of strongly coupled plasmas. At low densities $\left(N_{e}<10^{22} \mathrm{~m}^{-3}\right)$ ion dynamics correction is required, while at high density the motion of 


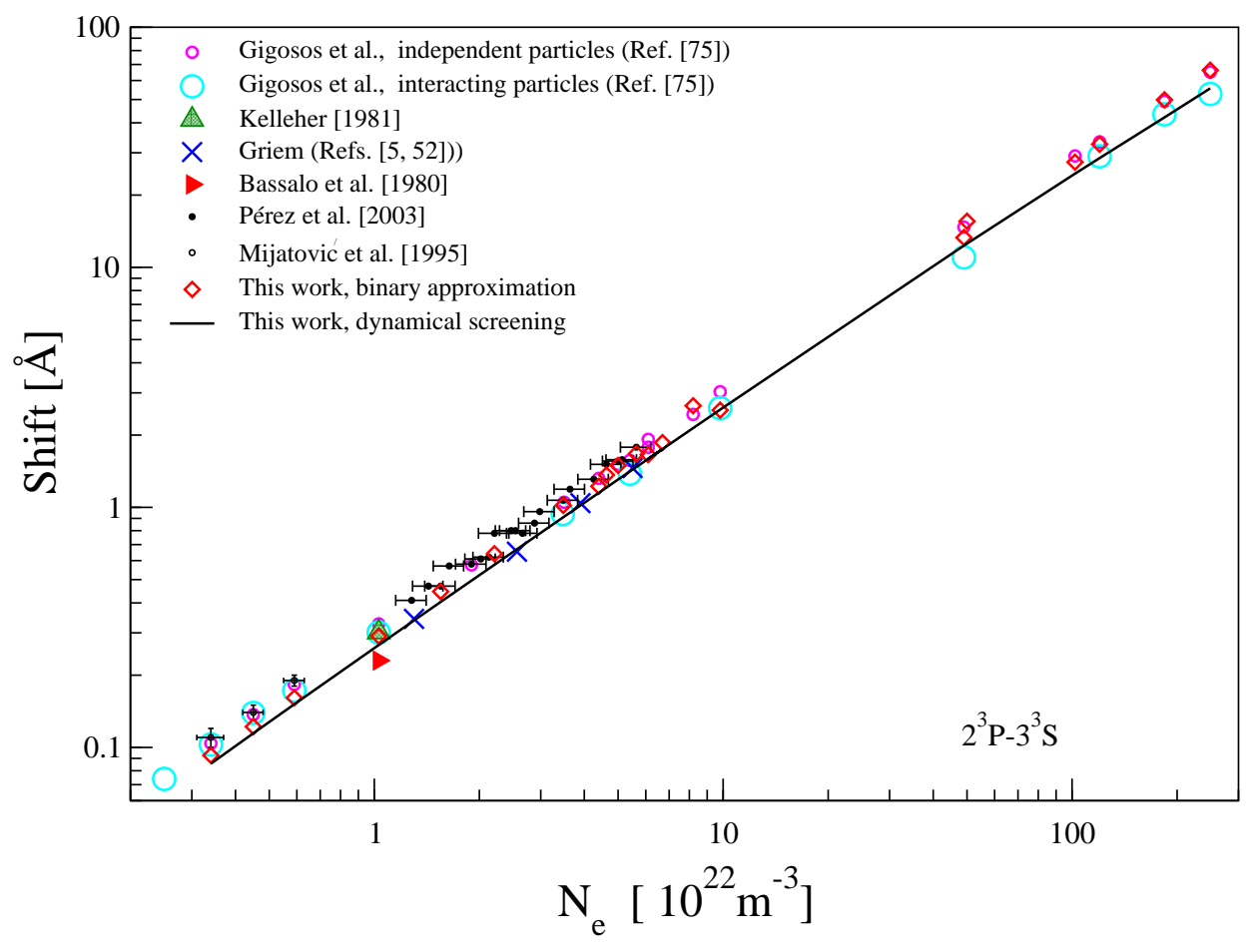

FIG. 7: (color online) The shift of the He I line $7065 \AA$ versus electron density. Comparison is made with available theoretical and experimental data. The electron temperature is taken according to the references $[5,27,30,32,52,75,76]$, see also Tabs. II and IV.

the ions is not significant [30]. Furthermore, a microscopic description of strong collisions via partial summation of the corresponding three-particle T-matrix [57] or the close-coupling method by Schöning [70] should be used to avoid artificial cut-off parameters. This is the object of future work.

\section{ACKNOWLEDGMENTS}

The authors express appreciation to Professor H. R. Griem for the assistance and to Professor M. A. Gonzalez for valuable discussions. This work was supported by the Deutsche Forschungsgemeinschaft within Graduiertenkolleg 567, Sonderforschungsbereich 198 and 652.

[1] H. R. Griem, Plasma Spectroscopy (McGraw-Hill, New York, 1964).

[2] W. Lochte-Holtgreven, Plasma Diagnostics (American Institute of Physics, New York, 1995).

[3] C. Pérez, I. de la Rosa, A. M. de Frutos, and S. Mar, Phys. Rev. A 44, 6785 (1991).

[4] C. Pérez, J. A. Aparicio, I. de la Rosa, S. Mar, and M. A. Gigosos, Phys. Rev. E 51, 3764 (1995).

[5] C. Pérez, R. Santamarta, M.I. de la Rosa, and S. Mar, Eur. Phys. J. D 27, 73 (2003).

[6] W. T. Chiang, D. P. Murphy, Y. G. Chen, and H. R. Griem, Z. Naturforsch 32 a, 818 (1977).

[7] V. Milosavljević and S. Djeniže, Astronomy and Astrophysics 393, 721 (2002).

[8] A. Beauchamp, F. Wesemael, and P. Bergeron, Astronomical Journal Supplement Series, 108, 559 (1997).

[9] M. S. Dimitrijević and S. Sahal-Bréchot, Astron. Astrophys. 136, 289 (1984).

[10] M. S. Dimitrijević, Astron. Astrophys. 112, 251 (1982).

[11] D. Branch, S. Benetti, D. Kasen, E. Baron, D. J. Jeffery, K. Hatano, R. A. Stathakis, Alexei V. Filippenko, Thomas Matheson, A. Pastorello, G. Altavilla, E. Cappellaro, L. Rizzi, M. Turatto, Weidong Li, Douglas C. Leonard, and Joseph C. Shields, Astrophys. J. 566, 1005 (2002).

[12] C. Fransson, R. A. Chevalier, A. V. Filippenko, B. Leibundgut, A. J. Barth, R. A. Fesen, R. P. Kirshner, D. C. Leonard, W. Li, P. Lundqvist, J. Sollerman, and S. D. Van Dyk, Astrophys. J. 572, 350 (2002). 


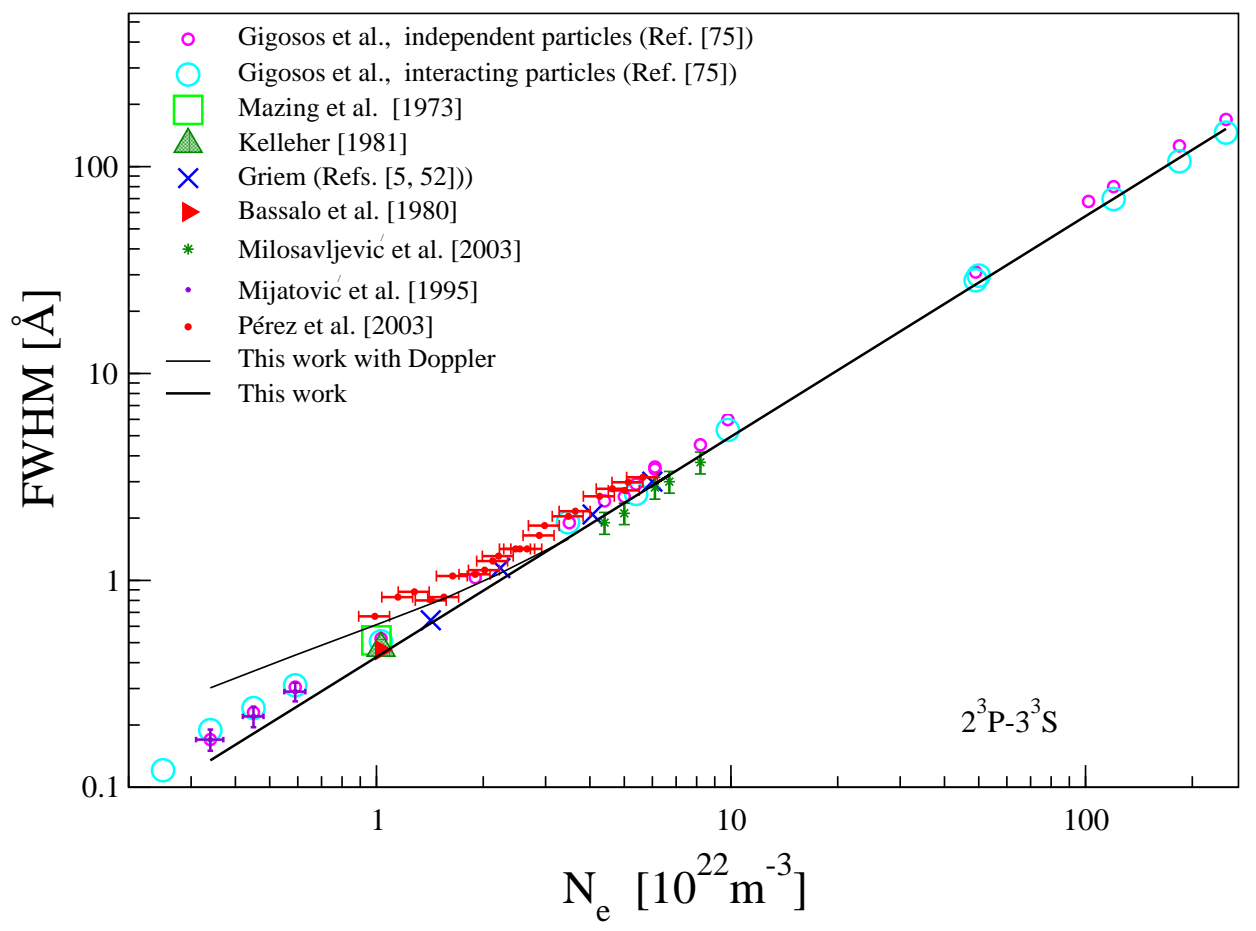

FIG. 8: (color online) The full width at half maximum of the He I line $7065 \AA$ versus electron density. Comparison is made with available theoretical and experimental data. The electron temperature is taken according to the references $[5,27,30,32,52,75-$ 77], see also Sec. 3, Tab. III.

[13] N. A. Webb, T.Naylor, and R. D. Jeffries, Astrophys. J. 568, L45 (2002).

[14] J. A. Harvin, D. R. Gies, W. G. Jr. Bagnuolo, L. R. Penny, and M. L. Thaller, Astrophys. J. 565, 1216 (2002).

[15] C. S. Jeffery, J. S. Drilling, P. M. Harrison, U. Heber, and S. Moehler, Astron. Astrophys. 125, 501 (1997).

[16] C. Rossi, R. F. Viotti, Th. Gäng, G. Bonanno, P. Bruno, A. Cali, R. Cosention, S. Scuderi, M. C. Timpanaro, and S. Desidera, in Proceeding of the conference "P Cygni 2000, 400 years of progress" Armagh 21-23 August 2000. ASP Conference Series, Vol. 233, edited by C. Sterken and M. de Groot (2001).

[17] E. L. Robinson, I. I. Ivans, and W. F. Welsh, Astrophysical Journal 565, 1169 (2002).

[18] J. -C. Gauthier, J. -P. Geindre, C. Goldbach, N. Grandjouan A. Mazure, and G. Nollez J. Phys. B: At. Mol. Phys. 14, 2099 (1981).

[19] T. Wilhein, D. Altenbernd, U. Teubner, E. Förster, R. Häßner, W. Theobald, and R. Sauerbrey, J. Opt. Soc. Am. B 15, 1235 (1998).

[20] U. Andiel, K. Eidmann, K. Witte, R. Mancini, and P. Hakel, Journal of Modern Optics 49, 2615 (2002).

[21] B. T. Vujičić, S. Djurović, and J. Halenka, Z. Phys. D 11, 119 (1989).

[22] B. Ya'akobi, E. V. George, G. Bekefi, and R. J. Hawryluk, J. Phys. B: At. Mol. Phys. 5, 1017 (1972).

[23] M. A. Gigosos, M. A. Gonzalez, B. Talin, and A. Calisiti, in Proceeding of the 17th International Conference on Spectral Line Shapes, edited by E. Dalimier (Frontier Group, Paris 2004) p. 451.

[24] A. Y. Potekhin, G. Chabrier, and D. Gilles, Phys. Rev. E 65, 36412 (2002).

[25] N. Konjević, A. Lesage, J. R. Fuhr, and W. L. Wiese, J. Phys. Chem. Ref. Data 31, 819 (2005).

[26] N. Konjević, A. Lesage, J. R. Fuhr, and W. L. Wiese, J. Phys. Chem. Ref. Data 31, 819 (2002).

[27] Z. Mijatović, N. Konjević, M. Ivković, and R. Kobilarov, Phys. Rev. E 51, 4891 (1995).

[28] S. Djeniže, Lj. Skuljan, and R. Konjević, J. Quant. Spectrosc. Radiat. Transf. 54, 581 (1995).

[29] H. F. Berg, A. W. Ali, R. Lincke, and H. R. Griem, Phys. Rev. 125, 199 (1962).

[30] D. E. Kelleher, J. Quant. Spec. Radiat. Transf. 25, 191 (1981).

[31] V. Milosavljević and S. Djeniže, New Astronomy 7, 543 (2002).

[32] V. Milosavljević and S. Djeniže, Eur. Phys. J. D 23, 385 (2003).

[33] A. Sreckovic, S. Bukvic and S. Djeniže, Eur. Phys. J. D 30, 93 (2004).

[34] S. Büscher, S. Glenzer, Th. Wrubel, and H. -J. Kunze, J. Quant. Spec. Radiat. Transf. 54, 73 (1995).

[35] A. G. Frank, V. P. Gavrilenko, and N. P. Kyrie, 16th International Conference on Spectral Line Shapes, Ed. Ch. A. Back (Melville, New York, 2002) p. 421.

[36] H. G. Adler and A. Piel, J. Quant. Spec. Radiat. Transf. 45, 11 (1991). 


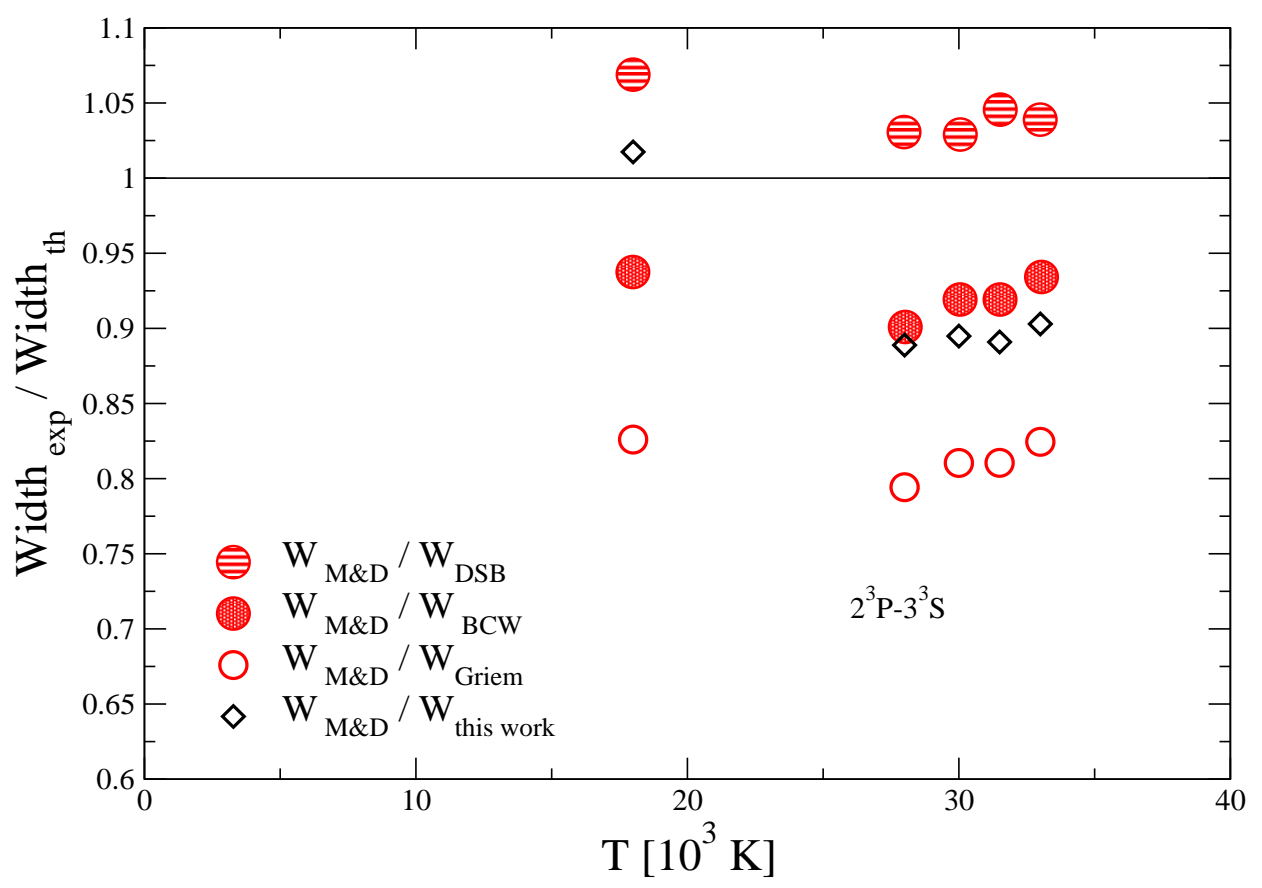

FIG. 9: (color online) Ratio of the experimental Stark FWHM of the He I line $7065 \AA$ to the various theoretical approaches versus corresponding experimental temperature from Milosavljević and Djeniže [32].

[37] V. Milosavljevic and S. Djeniže, Eur. Phys. J. D 15, 99 (2001).

[38] D. J. Heading, J. P. Marangos, and D. D. Burgess, J. Phys. B: At. Mol. Opt. Phys. 25, 4745 (1992).

[39] H. Suemitsu, K. Iwaki, Y. Takemoto, and E. Yoshida, J. Phys. B: At. Mol. Opt. Phys. 23, 1129 (1990).

[40] R. Kobilarov, N. Konjevic, and M. V. Popovic, Phys. Rev. A 40, 3871 (1989).

[41] Y. Guimeráns, E. J. Iglesias, D. Mandelbaum, and A. Sánchez, J. Quant. Spec. Radiat. Transf. 42, 39 (1989).

[42] J. R. Greig and L. A. Jones, Phys. Rev. a 1, 1261 (1970).

[43] D. D. Burgess and C. J. Cairns, J. Phys. B: At. Mol. Phys. 4, 1364 (1971).

[44] J. W. Birkeland, M. E. Bacon, and W. G. Braun, Phys. Rev. A 3, 354 (1971).

[45] R. N. Morris and J. Cooper, Can. J. Phys. 51, 1746 (1971).

[46] J. E. Jenkins and D. D. Burgess, J. Phys. B: At. Mol. Phys. 4, 1353 (1971).

[47] G. Röpke, T. Seifert, and K. Kilimann, Ann. Phys. (N.Y.)38, 381 (1981).

[48] S. Günter, Contrib. Plasma Phys. 29, 479 (1989).

[49] J. M. Bassalo, M. Cattani, and V. S. Walder, J. Quant. Spec. Radiat. Transf. 28, 75 (1982).

[50] M. S. Dimitrijević and S. Sahal-Bréchot, J. Quant. Spec. Radiat. Transf. 31, 301 (1984).

[51] H. R. Griem, M. Baranger, A. C. Kolb, and G. Oertel, Phys. Rev. 125, 177 (1962).

[52] H. R. Griem, Spectral Line Broadening by Plasmas (Academic Press, New York, 1974).

[53] M. A. Gonzalez (private communication).

[54] A. Brissaud, C. Goldbach, J. Leorat, A. Mazure, and G. Nollez, J. Phys. B 9, 1147 (1976).

[55] A. Calisti, R. Stamm, and B. Talin, Phys. Rev. A 38, 4883 (1988).

[56] S. Günter, L. Hitzschke, and G. Röpke, Phys. Rev. A 44, 6834 (1991).

[57] S. Günter, IPP MPI Report No. 5/67 (unpublished).

[58] S. Böddeker, S. Günter, A. Könies, L. Hitzschke, and H.-J. Kunze, Phys. Rev. E 47, 2785 (1993).

[59] A. Döhrn, P. Nowack, A. Könies, S. Günter, and V. Helbig, Phys. Rev. E 53, 6389 (1996).

[60] S. Günter and A. Könies, Phys. Rev. E 55, 907 (1997).

[61] W. Kraeft, D. Kremp, W. Ebeling, and G. Röpke, Quantum Statistics of Charged Particle Systems (Akademie-Verlag, Berlin, 1986).

[62] L. Hitzschke, G. Röpke, T. Seifert, and R. Zimmermann, J. Phys. B: At. Mol. Phys. 19, 2443 (1986).

[63] S. Sorge, A. Wierling, G. Röpke, W. Theobald, R. Sauerbrey, and T. Wilhein, J. Phys. B: At. Mol. Opt. Phys. 33, 2983 (2000).

[64] C. F. Jr. Hooper, Phys. Rev. 165, 215 (1968).

[65] S. Günter, A. Könies, J. Quant. Spec. Radiat. Transf. 52, 819 (1994).

[66] L. Hitzschke and S. Günter, J. Quant. Spectrosc. Radiat. Transf. 56, 423 (1996).

[67] D. R. Bates and A. Damgaard, Phil. Trans. Roy. Soc. London, A 242, 101 (1949). 


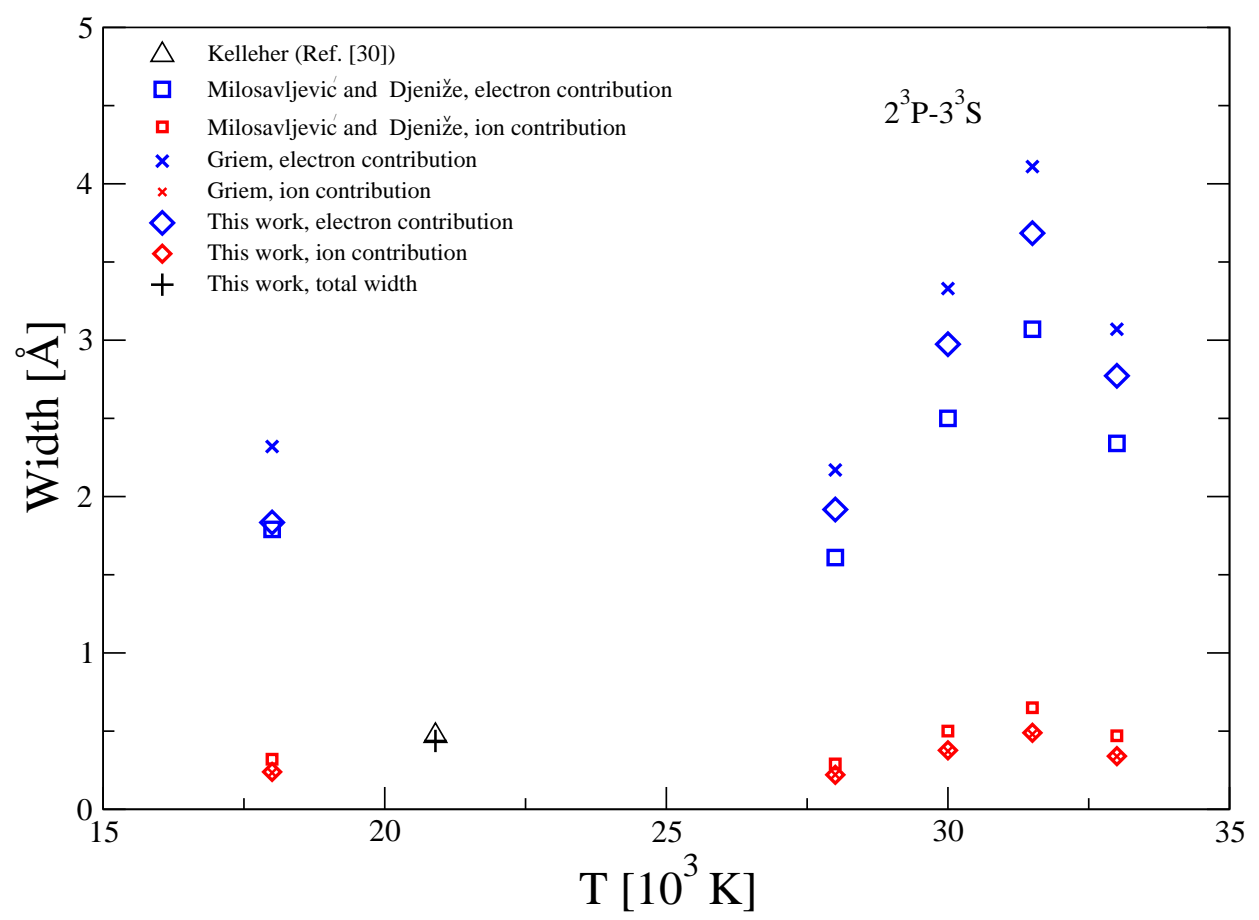

FIG. 10: (color online) The electronic and ionic width contributions of the He I line $7065 \AA$ as a function of temperature. Comparison is made with theoretical and experimental data [32].

[68] I. I. Sobelman. Atomic Spectra and Radiative Transitions (Springer-Verlag, Berlin, 1992).

[69] H. R. Griem, Phys. Rev. 128, 515 (1962).

[70] T. Schöning, J. Phys. B: At. Mol. Opt. Phys. 26, 899 (1993).

[71] G. Röpke, S. Sorge A. Wierling, Contrib. Plasma Phys. 41, 187 (2001).

[72] S. Sorge, S. Günter and G. Röpke, J. Phys. B: At. Mol. Phys. 32, 675 (1999).

[73] H. A. Bethe and E. E. Salpeter, Quantum Mechanics of One- and Two-Electron Atoms (Plenum Publishing Corporation, New York, 1977).

[74] J. Halenka. Z. Phys. D 16, 1 (1990).

[75] M. A. Gigosos et al. (unpublished).

[76] J. M. Bassalo, M. Cattani, and V. S. Walder, Phys. Rev. A 22, 1194 (1980)

[77] M. A. Mazing and V. A. Selemzin, Sov. Phys. Lebedev Inst. Rep. 4, 42 (1973).

[78] G. Röpke, H. Reinholz, C. Neissner, B. Omar, and A. Sengebusch, Contrib. Plasma Phys. 45, 414 (2005).

[79] B. Omar, A. Wierling, S. Günter, and G. Röpke, in Proceedings of the 17th International Conference on Spectral Line Shapes, edited by E. Dalimier (Frontier Group, Paris 2004) p. 150.

[80] C. S. Diatta, thesis, University d'Orleans (1977).

[81] M. S. Dimitrijević and S. Sahal-Bréchot, Astron. Astrophys. Suppl. Ser. 82, 519 (1990).

[82] J. Seidel, Z. Naturf. 34a, 1385 (1979).

[83] J. Barnard, J. Cooper and E. W. Smith, J. Quant. Spec. Radiat. Transf. 14, 1025 (1974).

[84] C. A. Iglesias, Phys. Rev. A 27, 2705 (1983).

[85] C. A. Iglesias, J. L. Lebowitz, D. MacGowan, Phys. Rev. A 28, 1667 (1983). 


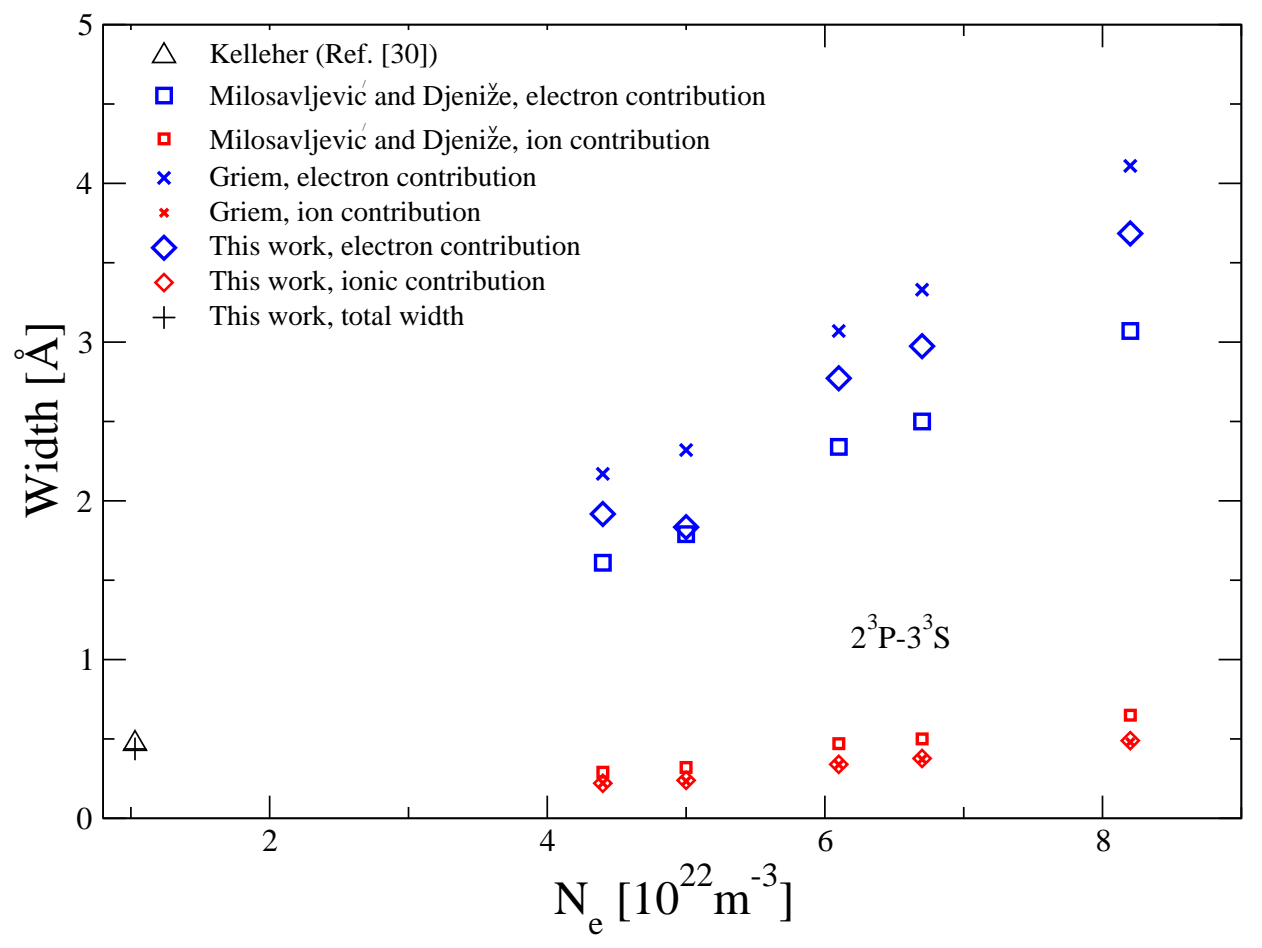

FIG. 11: (color online) The electronic and ionic width contributions of the He I line $7065 \AA$ versus electron density. Comparison is made with theoretical and experimental data [32].

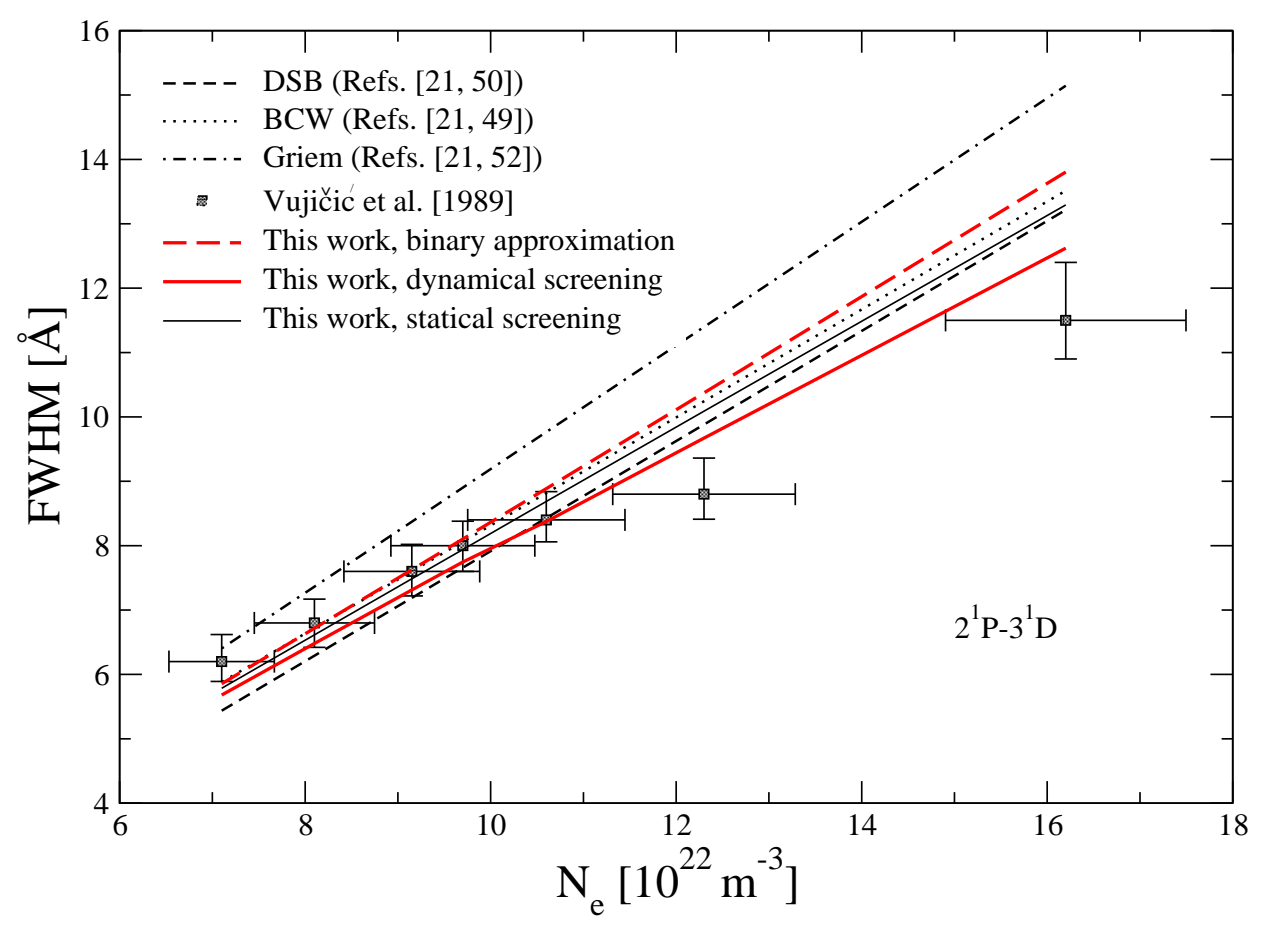

FIG. 12: (color online) The full width at half maximum of the He I line $6678 \AA$ versus electron density. Comparisons are made with the experimental data of Vujičić et al. [21], the calculated theoretical values given in the same reference and our data at an electron temperature $3 \times 10^{4} \mathrm{~K}$. 


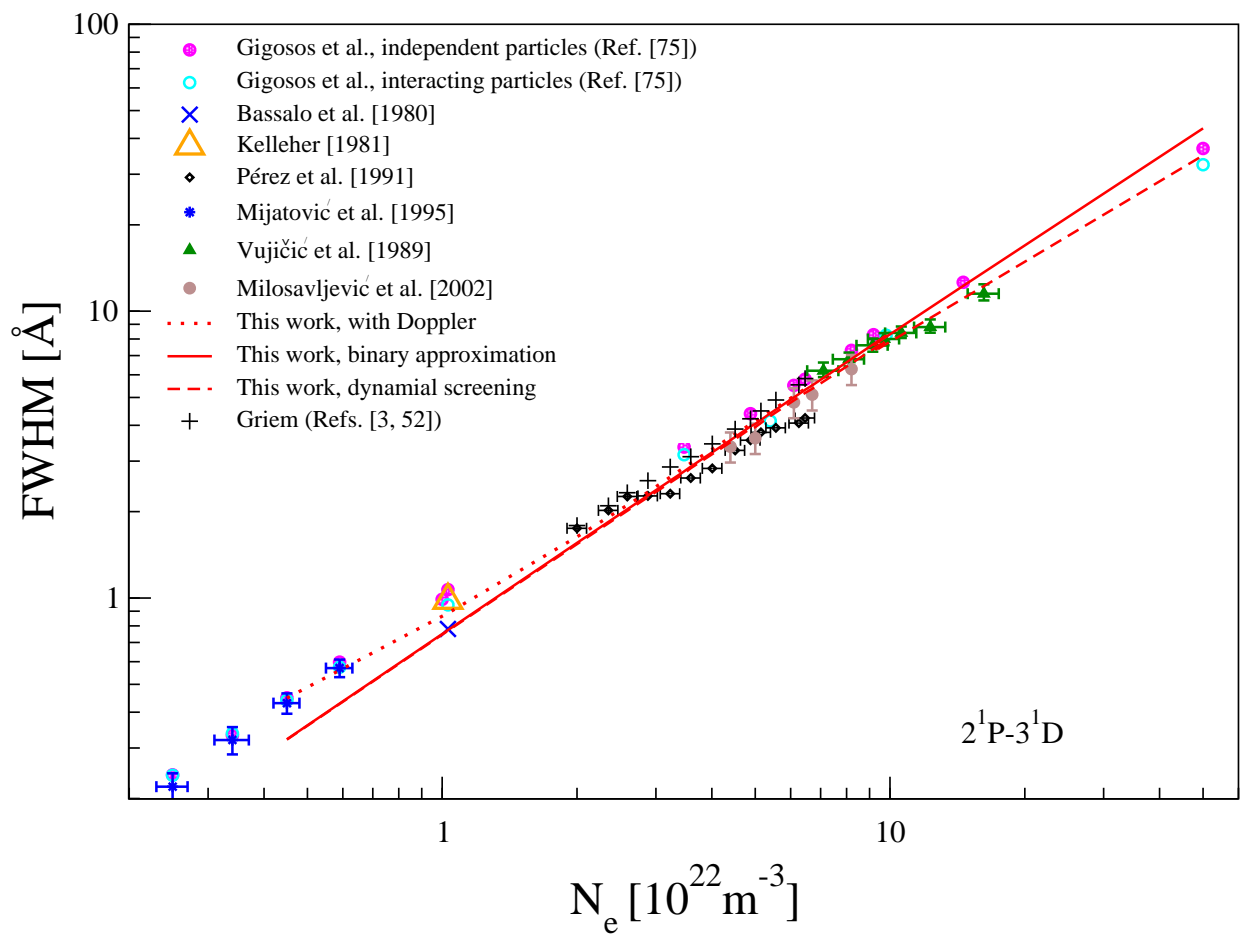

FIG. 13: (color online) The full width at half maximum of the He I line $6678 \AA$ versus electron density, with and without Doppler broadening, dynamical screening is included. The electron temperature is taken according to the references $[3,7,21$, $27,30,75,76]$, see also Tab. III.

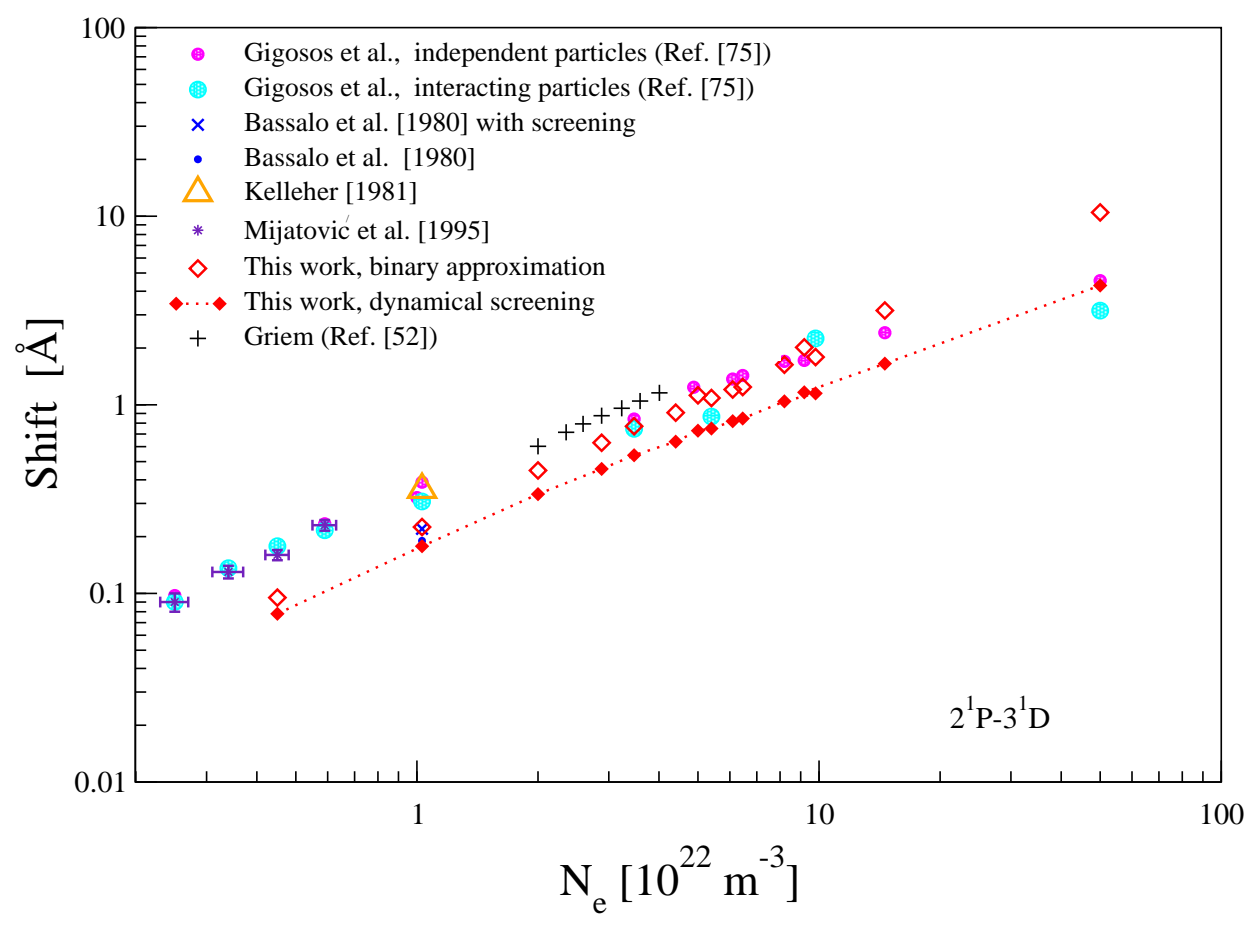

FIG. 14: (color online) The shift of the He I line $6678 \AA$ versus electron density. Comparison is made with available theoretical and experimental data taking the corresponding electron temperatures [27, 30, 75, 76], see also Tab. II. 


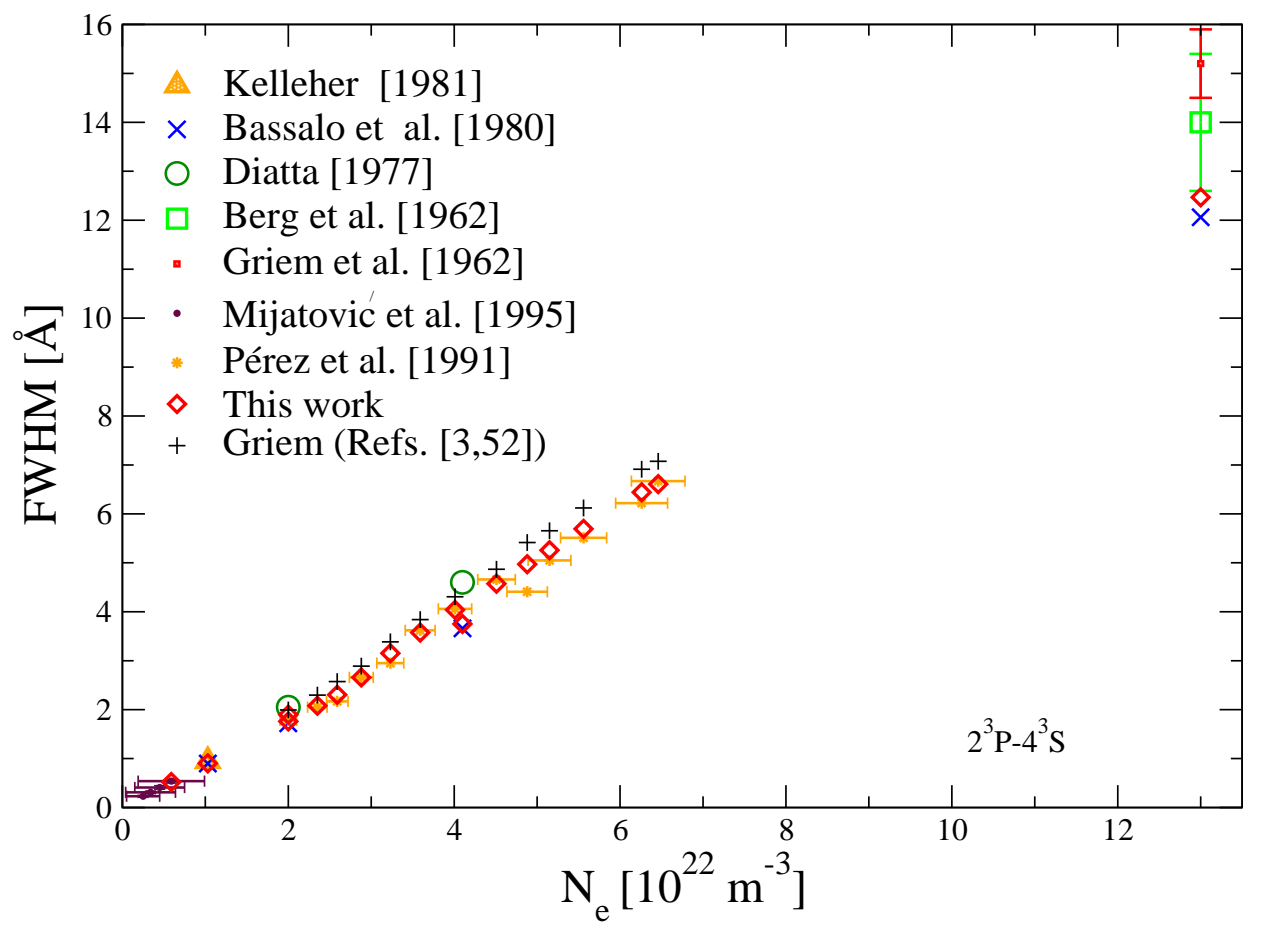

FIG. 15: (color online) The full width at half maximum of the He I line $4713 \AA$ as a function of electron density. Available theoretical and experimental data are included. The electron temperature is taken according to the references $[3,27,29,30$, $76,80]$, see also Tab. III.

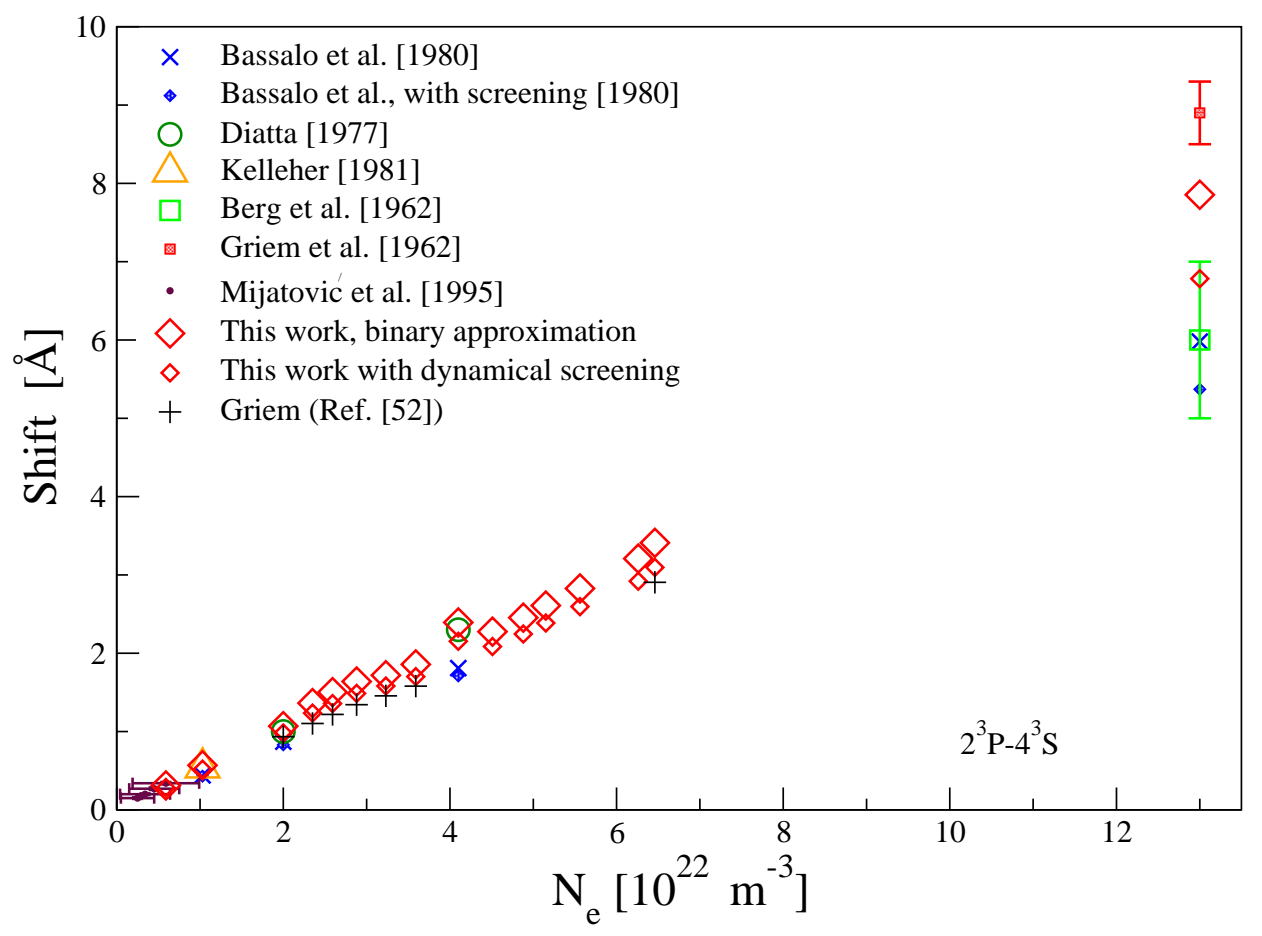

FIG. 16: (color online) The shift of the He I line $4713 \AA$ as a function of electron density. Comparison is made with previous theoretical and experimental data. The electron temperature is taken according to the references $[27,29,30,76,80]$, see also Tab. II. 


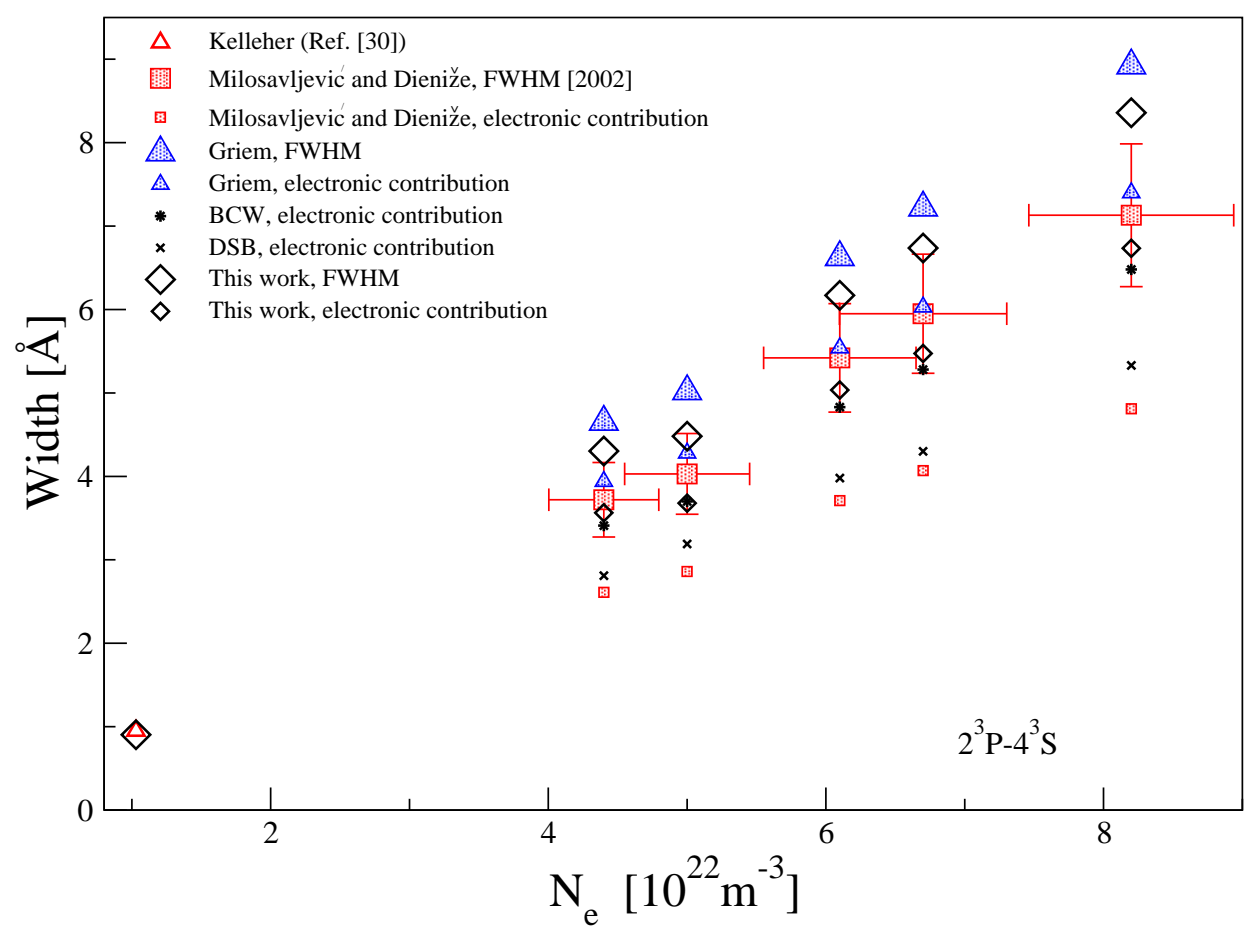

FIG. 17: (color online) Stark width versus electron density of the He I line $4713 \AA$. The experimental data of Milosavljević and Djeniže [31], the theoretical data of Griem [52], BCW [49], and DSB [81] reported from the same Ref. [31], are compared with our calculations.

\begin{tabular}{|c|c|c|c|c|c|c|}
\hline$\lambda(\AA)$ & $N_{\mathrm{e}}\left(10^{22} \mathrm{~m}^{-3}\right)$ & $T_{e}\left(10^{3} \mathrm{~K}\right)$ & $d_{\mathrm{B}}(\AA)$ & $d_{\mathrm{G}}$ & $d_{\mathrm{th}}(\AA)$ & $d_{\exp }(\AA)$ \\
\hline \multirow[t]{4}{*}{7281} & 0.6 & 4.0 & - & - & $0.208 / 0.193$ & $0.16 \pm 15 \%[28]$ \\
\hline & 1.03 & 20.9 & $0.37 / 0.36$ & $0.528 / 0.480$ & $0.438 / 0.404$ & $0.473[30]$ \\
\hline & 3.469 & 20.0 & - & $1.66 / 1.49$ & $1.552 / 1.42$ & $1.48[4]$ \\
\hline & 9.118 & 20.0 & - & $4.34 /-$ & $4.212 / 3.793$ & $4.30[4]$ \\
\hline \multirow[t]{4}{*}{7065} & 0.45 & 22.7 & - & $0.137 / 0.139$ & $0.122 / 0.112$ & $0.14[27]$ \\
\hline & 1.03 & 20.9 & $0.23 / 0.23$ & $0.327 / 0.301$ & $0.291 / 0.265$ & $0.302[30]$ \\
\hline & 3.48 & 20.0 & - & $1.91 / 0.93$ & $1.02 / 0.928$ & $1.07[5]$ \\
\hline & 249.0 & 63.476 & - & $65.0 / 52.7$ & $66.25 / 55.57$ & - \\
\hline \multirow[t]{4}{*}{6678} & 1.03 & 20.9 & $0.22 / 0.19$ & $0.389 / 0.307$ & $0.225 / 0.178$ & $0.355[30]$ \\
\hline & 4.88 & 42.05 & - & $1.24 /-$ & $0.91 / 0.66$ & - \\
\hline & 6.46 & 36.45 & - & $1.43 /-$ & $1.242 / 0.846$ & - \\
\hline & 8.2 & 31.5 & - & $1.7 /-$ & $1.63 / 1.04$ & - \\
\hline \multirow[t]{5}{*}{4713} & 0.59 & 23.6 & - & - & $0.31 / 0.286$ & $0.34 \pm 0.015[27]$ \\
\hline & 1.03 & 20.9 & $0.44 / 0.43$ & - & $0.569 / 0.52$ & $0.538[30]$ \\
\hline & 2.0 & 18.0 & $0.87 / 0.83$ & - & $1.07 / 0.982$ & $1.0[80]$ \\
\hline & 4.1 & 20.0 & $1.81 / 1.71$ & - & $2.393 / 2.153$ & $2.3[80]$ \\
\hline & 13.0 & 20.0 & $5.98 / 5.37$ & - & $7.853 / 6.783$ & $6.0 \pm 1.0[29]$ \\
\hline
\end{tabular}

TABLE II: Comparison of the calculated shift (values without/with screening) $d_{\mathrm{B}}$ by Bassalo et al. [76], $d_{\mathrm{G}}$ from molecular dynamic simulation by Gigosos et al. [75] for independent and interacting particles, our calculation $d_{\mathrm{th}}$ and the experimental results $d_{\exp }$ have been taken from Refs. [4, 5, 27-30, 80]. 


\begin{tabular}{|c|c|c|c|c|c|c|}
\hline$\lambda(\AA)$ & $N_{\mathrm{e}}\left(10^{22} \mathrm{~m}^{-3}\right)$ & $T_{e}\left(10^{3} \mathrm{~K}\right)$ & $W_{\mathrm{B}}(\AA)$ & $W_{\mathrm{G}}$ & $W_{\text {th }}(\AA)$ & $W_{\exp }(\AA)$ \\
\hline \multirow[t]{3}{*}{7281} & 1.03 & 20.9 & $0.8 / 0.8$ & $0.929 / 0.90$ & $0.813 / 0.813$ & $0.88[30]$ \\
\hline & 3.469 & 20.0 & - & $3.20 / 2.97$ & $2.795 / 2.794$ & $3.84[4]$ \\
\hline & 9.188 & 20.0 & - & $8.43 /-$ & $7.602 / 7.596$ & $9.24[4]$ \\
\hline \multirow[t]{5}{*}{7065} & 0.45 & 22.7 & - & $0.23 / 0.241$ & $0.193 / 0.193$ & $0.22[27]$ \\
\hline & 1.03 & 20.9 & $0.46 / 0.46$ & $0.523 / 0.507$ & $0.437 / 0.437$ & $0.47[30]$ \\
\hline & 3.48 & 20.0 & - & $1.90 / 1.91$ & $1.497 / 1.497$ & $2.04[5]$ \\
\hline & 6.1 & 33.0 & - & $3.53 /-$ & $3.112 / 3.112$ & $2.81 \pm 0.3372[32]$ \\
\hline & 249.0 & 63.476 & - & 169.0/146.0 & $157.14 / 156.35$ & - \\
\hline \multirow[t]{4}{*}{6678} & 1.03 & 20.9 & $0.78 / 0.78$ & $1.07 / 0.946$ & $0.773 / 0.769$ & $0.98[30]$ \\
\hline & 4.88 & 42.05 & - & $4.39 /-$ & $3.963 / 3.895$ & $3.55[3]$ \\
\hline & 6.46 & 36.45 & - & $5.79 /-$ & $5.316 / 5.186$ & $4.24[3]$ \\
\hline & 8.2 & 31.5 & - & $7.3 /-$ & $6.81 / 6.57$ & $6.28[31]$ \\
\hline \multirow[t]{6}{*}{4713} & 0.59 & 23.6 & - & - & $0.521 / 0.521$ & $0.54 \pm 0.04[27]$ \\
\hline & 1.03 & 20.9 & $0.90 / 0.90$ & - & $0.904 / 0.904$ & $0.95[30]$ \\
\hline & 2.0 & 18.0 & $1.72 / 1.72$ & - & $1.898 / 1.898$ & $2.05[80]$ \\
\hline & 4.1 & 20.0 & $3.66 / 3.66$ & - & $3.752 / 3.75$ & $4.6[80]$ \\
\hline & 6.46 & 36.45 & - & - & $6.614 / 6.607$ & $6.67[3]$ \\
\hline & 13.0 & 20.0 & $12.06 / 12.06$ & - & $12.496 / 12.468$ & $14.0 \pm 1.4[29]$ \\
\hline
\end{tabular}

TABLE III: Comparison of the calculated FWHM (values without/with screening are given) $W_{\mathrm{B}}$ of Bassalo et al. [76], $W_{\mathrm{G}}$ from molecular dynamic simulation by Gigosos et al. [75] for independent and interacting particles, our calculation $W_{\text {th }}$ and the experimental values $W_{\exp }$ are given in Refs. [3-5, 27, 29-32, 80].

\begin{tabular}{|cccccc|}
\hline$N_{\mathrm{e}}\left(10^{22} \mathrm{~m}^{-3}\right)$ & $T_{e}\left(10^{3} \mathrm{~K}\right)$ & $d_{\mathrm{G}}(\AA)$ & $d_{1}(\AA)$ & $d_{2}(\AA)$ & $d_{3}(\AA)$ \\
\hline 9.8 & 42.0 & $3.03 / 2.59$ & 2.54 & 2.502 & 2.352 \\
49.0 & 44.561 & $14.7 / 11.0$ & 13.293 & 12.818 & 11.975 \\
50.0 & 20.0 & $15.52 / 12.8$ & 15.557 & 14.827 & 13.54 \\
102.0 & 51.988 & $29.1 /-$ & 27.425 & 26.229 & 24.078 \\
120.0 & 52.104 & $33.3 / 29.0$ & 32.558 & 30.857 & 28.332 \\
184.0 & 56.049 & $49.1 / 43.4$ & 49.845 & 46.67 & 42.453 \\
249.0 & 63.476 & $65.0 / 52.7$ & 66.245 & 61.496 & 55.566 \\
\hline
\end{tabular}

TABLE IV: Comparison of the calculated shift values of line (7065 ^) from molecular dynamic simulation by Gigosos et al. [75] for independent and interacting particles $d_{\mathrm{G}}$, our calculation for binary approximation $d_{1}$, by taking Debye screening cut-off parameter $d_{2}$ and in the case of dynamical screening $d_{3}$ are given. 